\title{
Double Dirichlet series over function fields
}

\author{
Benji Fisher and Solomon Friedberg
}

\begin{abstract}
We construct a finite-dimensional vector space of functions of two complex variables attached to a smooth algebraic curve $C$ over a finite field $\mathbb{F}_{q}, q$ odd, and a level. These functions collect the analytic information about the cohomology of the curve and its quadratic twists that is encoded in the corresponding $L$-functions; they are double Dirichlet series in two independent complex variables $s$ and $w$. We prove that these series satisfy a finite, non-abelian group of functional equations in the two complex variables $(s, w)$ and are rational functions in $q^{-s}$ and $q^{-w}$ with a specified denominator. The group is $D_{6}$, the dihedral group of order 12 .
\end{abstract}

\section{Introduction}

The aim of this paper is to exhibit a new, natural, finite-dimensional vector space of functions of two complex variables attached to a smooth algebraic curve $C$ over a finite field $\mathbb{F}_{q}, q$ odd, and a level (in a sense to be defined below). These functions collect the analytic information about the cohomology of the curve and its quadratic twists that is encoded in the corresponding $L$-functions. The functions are double Dirichlet series in two independent complex variables $s$ and $w$. We prove that these series satisfy a finite, non-abelian group of functional equations in the two complex variables $(s, w)$ and are rational functions in $q^{-s}$ and $q^{-w}$ with a specified denominator. The group is in fact $D_{6}$, the dihedral group of order 12 .

Let $C$ be a smooth projective curve over a finite field $\mathbb{F}_{q}, q$ odd. Let $K$ be the function field of $C$. Suppose that $\mathbb{F}_{q}$ is algebraically closed in $K$. We obtain the rational functions mentioned above by studying the family of quadratic twists of an arbitrary GL(1) (Hecke) $L$-function $L\left(s, \rho_{1}\right)$ defined over $K$. To study this family, we attach to each effective divisor $D$ on $C$, prime to a fixed sufficiently large finite set $S$ of places, a quadratic character $\chi_{D}$. Consistent with class field theory, this map is necessarily not canonical, but depends on choices of representatives for a certain ray class group modulo squares. Then we introduce a second complex variable $w$ and form the sum of $L$-series (initially defined for $\operatorname{Re}(s), \operatorname{Re}(w)>1$ )

$$
Z\left(s, w ; \rho_{1}, \rho_{2}\right)=\sum_{0 \leqslant D \in \operatorname{Div}(C-S)} \frac{L\left(s, \rho_{1} \chi_{D}\right) a\left(s, \rho_{1}, D\right) \rho_{2}(D)}{|D|^{w}} .
$$

Here $L\left(s, \rho_{1} \chi_{D}\right)$ is the twisted $L$-function with the primes in $S$ and in the conductor of $\chi_{D}$ removed; the sum is over effective divisors $D$ of $C$ prime to $S ;|D|^{w}=q^{(\operatorname{deg} D) w} ; \rho_{2}$ is another Hecke character; and the 'correction factor' $a\left(s, \rho_{1}, D\right)$ is a polynomial in $q^{-s}$ that is 1 if $D$ is square-free, i.e. if $\operatorname{ord}_{v}(D) \leqslant 1$ for all places $v$ of $C$. This correction factor arises in the calculation of the Fourier coefficients of Eisenstein series on the double cover of GL(2); its role in our arguments will be

Received 9 November 2001, accepted in final form 17 September 2002.

2000 Mathematics Subject Classification 11M38 (primary), 11G20, 11L05, 11L07, 11R47, 11R58, 14H05 (secondary).

Keywords: algebraic curve, $L$-function, quadratic twist, rational function, sum of $L$-functions.

Research supported by NSF grant DMS-9970118 (Friedberg).

This journal is (C) Foundation Compositio Mathematica 2004. 


\section{B. Fisher AND S. FriedBerG}

described below. For a complete definition see $\S 1$ below. Note that we use $|D|$ for what is sometimes denoted $N(D)$.

The sum of $L$-functions may also be expressed as a double Dirichlet series. We shall show in Lemma 3.1 that

$$
Z\left(s, w ; \rho_{1}, \rho_{2}\right)=L\left(2 s+2 w-1, \rho_{1}^{2} \rho_{2}^{2}\right) \sum_{D_{1}, D_{2}} \frac{\rho_{1}\left(D_{1}\right) \rho_{2}\left(D_{2}\right)}{\left|D_{1}\right|^{s}\left|D_{2}\right|^{w}} \chi_{D_{2}}\left(D_{1}\right),
$$

where the sum is over disjoint, effective divisors in $\operatorname{Div}(C-S)$, and the $L$-function once again has the Euler factors in $S$ removed.

Recall that the partial zeta-function of a curve is of the form $\zeta_{K, S}(s)=P\left(q^{-s}\right) /\left(1-q^{1-s}\right)$; here the polynomial $P$ is given by the characteristic polynomial of Frobenius acting on a first cohomology and 'partial' indicates that we have removed the Euler factors corresponding to the places in $S$. A non-trivial GL(1) partial $L$-function $L\left(s, \rho_{1}\right)$ is similarly a polynomial in $q^{-s}$. In studying such $L$-functions, it is common to restrict to quasicharacters $\rho_{1}$ that factor through the idèles of norm 1 (see [Wei74, p. 134]), since more general quasicharacters give shifts of these $L$-functions. In our work, however, it is helpful to weaken this restriction slightly, considering these functions with a shift in $s$ that corresponds to replacing $q^{-s}$ by $\omega q^{-s}$ where $\omega$ is an $n$th root of unity, for some fixed positive even $n$. We consider the vector space spanned by such $L$-functions as one varies $\rho_{1}$ and $\rho_{2}$ with fixed level $F$. This finite-dimensional vector space $V(F, n)$ does not depend on the choices of representatives used to define $\chi_{D}$ above: it is canonical (Proposition 1.2); see also Remark 1.3 for a definition of $V(F, n)$ which does not depend on choices. Then we show the main theorem of this paper (Theorem 4.1 below).

Theorem. Let $Z(s, w)$ be in $V(F, n)$. Then $Z(s, w)$ has meromorphic continuation to all $(s, w)$ in $\mathbb{C}^{2}$ and is a rational function in $q^{-s}$ and $q^{-w}$. In fact,

$$
\left(1-q^{n(1-s)}\right)\left(1-q^{n(1-w)}\right)\left(1-q^{n(3 / 2-s-w)}\right) Z(s, w)
$$

is a polynomial of degree at most $2 \operatorname{deg}(F)+2 n+4 g-4$ in each of $q^{-s}$ and $q^{-w}$, where $g$ is the genus of $C$.

Let us outline the proof. The meromorphic continuation is obtained by establishing two separate functional equations for the double Dirichlet series in $V(F, n)$. The first functional equation (Theorem 2.6) sends $(s, w) \rightarrow\left(1-s, s+w-\frac{1}{2}\right)$. This is obtained from the functional equations for the $L$-functions in the numerators in the sum (0.1). The main ingredient here is control of the epsilon-factors that arise in such a functional equation (Lemma 2.2). (Since the numerators have analytic continuations, this also enlarges the region on which $Z(s, w)$ is defined.) The second functional equation sends $(s, w) \rightarrow(w, s)$. This is obtained by an interchange of summation and by using quadratic reciprocity; compare (0.2). In essence this second functional equation states that the double Dirichlet series, originally a sum of Euler products in $s$ with functional equation, is also a sum of Euler products in $w$ with functional equation! This is not true unless one modifies the $L$-series slightly; this is the role of the factors $a\left(s, \rho_{1}, D\right)$ introduced above. In fact, Bump, Friedberg, and Hoffstein [BFH04] have shown that the correction factors $a\left(s, \rho_{1}, D\right)$ are uniquely determined by this condition. Once we have these functional equations, we use them to obtain continuation to a larger region whose convex hull is all of $\mathbb{C}^{2}$. Then invoking the convexity principle for tube domains, the meromorphicity follows. The rationality then follows by a variation on the one-variable argument.

There is a subtle point here: In order to achieve the symmetry $(s, w) \rightarrow(w, s)$, we must define a quadratic character $\chi_{D}$ of $\operatorname{Div}(C)$ for each divisor $D$ of $C$ prime to $S$; however, such quadratic characters correspond naturally to elements $a \in K^{\times} /\left(K^{\times}\right)^{2}$ (they are given by the quadratic residue symbols). Thus this difficulty is analogous to extending results in algebraic number theory from class number one number fields to the general case. In the present situation, though the individual 


\section{Double Dirichlet SERIES OVER FUNCTION FIELDS}

$L$-functions are obtained from adelic objects, $Z(s, w)$ is an infinite sum of such, and such a difficulty appears intrinsic to the situation. We solve this problem by choosing a coset representative and a quadratic character for each element of the ray class group modulo squares. With these choices, we can define $\chi_{D}$ for all $D$ (see Equation (1.1) and Lemma 1.1 below for details). As mentioned above, though an individual sum of the form (0.1) depends on these choices, the span of such sums for fixed level does not.

As a consequence of the main theorem above, one can control the size of sums of $L$-functions, simply by studying the coefficients of the resulting rational function. For example, fix a congruence class $j \bmod n$. We show in Proposition 5.1 that for each pair $\left(\rho_{1}, \rho_{2}\right)$ as above there are certain rational functions $C_{j}(s), D_{j}(s)$ in $q^{-s}$, determined from $Z\left(s, w ; \rho_{1}, \rho_{2}\right)$, such that for $R \equiv j \bmod n$, $R \gg 0$,

$$
\sum_{\substack{0 \leqslant D \in \operatorname{Div}(C-S) \\ \operatorname{deg}(D)=R}} L\left(s, \rho_{1} \chi_{D}\right) a\left(s, \rho_{1}, D\right) \rho_{2}(D)=C_{j}(s) q^{R}+D_{j}(s)\left(q^{3 / 2-s}\right)^{R} .
$$

In particular, for $\operatorname{Re}(s)>1 / 2$ the sum of $L$-functions times correction factors grows like $C_{j}(s) q^{R}$ as $R \rightarrow \infty$ in the arithmetic progression $R \equiv j \bmod n$, provided $C_{j}(s) \neq 0$.

Let us put this work in context. In the rational number field case, Siegel [Sie56] observed that a sum of quadratic $L$-functions, which is roughly of the form $\sum L\left(z, \chi_{m}\right) a(s, m)|m|^{-s}$, may be obtained by taking the Mellin transform of a truncated half-integral weight Eisenstein series in $s$. In fact, Siegel writes that this series is

eine meromorphe Funktion der zwei Veränderlichen $s, z$, welche für $z=0$ in $\zeta(s)$ übergeht und sowohl in $s$ als auch in $z$ je einer Funktionalgleichung genügt.

The present paper may be regarded as further development of this viewpoint; see also Bump, Friedberg, and Hoffstein [BFH96]. In a pair of important papers, Goldfeld and Hoffstein [GH85] and Goldfeld, Hoffstein, and Patterson [GHP82] used the theory of half-integral weight Eisenstein series over the rationals (respectively over a totally imaginary number field of class number one) along with sieve methods and the study of hypergeometric functions to estimate the average of twisted $L$-values over fundamental discriminants. In the number field case we know of no work estimating the analogous sum for a general number field. However, the present paper indicates the natural way to formulate this problem for arbitrary class number, and either metaplectic Eisenstein series or the methods of this paper could be used to give such estimates.

Sums of quadratic twists of the zeta-function for the rational function field $K=\mathbb{F}_{q}(T)$ with $q \equiv$ 1 mod 4 were considered by Hoffstein and Rosen [HR92], once again using the theory of metaplectic Eisenstein series. For this situation, all relevant epsilon-factors are 1; see Remark 2.1. However, they stopped short of showing that the series so obtained is a rational function in two variables. (In fact, their method produced extraneous poles; compare their Proposition 4.1 to the calculation in $\S 6$ below.) The Mellin transform of one metaplectic Eisenstein series for such $K$ was noted to be rational in [Hof93]. As outlined above, our methods are different: we construct the group of functional equations directly, avoiding the use of metaplectic Eisenstein series. In our work, the functional equation obtained in [HR92, Hof93] by the Mellin transform and the automorphic properties of the Eisenstein series is a consequence of a functional equation obtained simply by an interchange of the order of summation. More precisely, the methods based on metaplectic Eisenstein series give a group of four functional equations which is a subgroup of the group of 12 functional equations we present here (see [Hof93, pp. 84-85]). The method of generating functional equations for double Dirichlet series by an interchange of summation was introduced in [BFH96, pp. 167-168]. The approach outlined there may be used to establish a subgroup of six functional equations in our instance, but does not give the symmetry $(s, w) \rightarrow(w, s)$. 


\section{B. Fisher AND S. FriedberG}

It would be interesting to have an intrinsic geometric interpretation for the series $Z(s, w)$, but the approaches to date are analytic and do not yield this. Also, as Goldfeld and Hoffstein already suggest for the number field case [GH85], it is natural to look at twists of higher-rank objects. See Bump, Friedberg, and Hoffstein [BFH90a, BFH90b, BFH96, FH95] for some results and a further development of this theme. Analogously to the results in this paper, one expects sums of the quadratic twists $L\left(s, \pi \otimes \chi_{D}\right)$ in the function field case to be rational functions for $\pi$ automorphic on GL $(r), r \leqslant 3$. We will present such results separately (there is an extra symmetry which we establish in the present case that is not available in the others; note that the correction factors for GL(3) have only recently been written down [BFH04]). Included here are sums involving more than two variables, provided the total degree of the $L$-functions being summed is at most 3 . Higher-order twists can probably also be summed to give rational functions, at least for rank 1 ( $n$ th-order twists) and rank 2 (cubic twists), cf. [FHL03, Lie94].

The remainder of this paper is organized as follows. Section 1 introduces the notation and defines the vector space of double sums we are considering. This vector space is also shown to be canonical. Section 2 gives the first functional equation, Theorem 2.6, and $\S 3$ the second, Theorem 3.3. Section 4 gives the proof of the main result, Theorem 4.1. In $\S 5$ we use this to estimate the size of sums of $L$-functions. Finally, $\S 6$ presents two examples: we compute the sums explicitly in low level when $C=\mathbb{P}^{1}$ and when $C$ is the elliptic curve $y^{2}=x^{3}-x-1$ over $\mathbb{F}_{3}$.

\section{Definition of the double Dirichlet series}

Throughout the remainder of this paper, let $C$ be a smooth projective curve over a finite field $\mathbb{F}_{q}$, $q$ odd. Let $K$ be the function field of $C$, and assume that $\mathbb{F}_{q}$ is algebraically closed in $K$.

The double Dirichlet series is a sum of GL(1) automorphic $L$-series. Let us recast these objects in the language of divisors. Let $\operatorname{Div}(C)$ be the group of $\mathbb{F}_{q}$-rational divisors on $C$, deg the degree map deg $: \operatorname{Div}(C) \rightarrow \mathbb{Z}, \operatorname{Div}_{0}(C)$ the divisors of degree 0, $\operatorname{Div}_{P}(C)$ the subgroup of $\operatorname{Div}_{0}(C)$ consisting of the principal divisors $(f), f \in K^{\times}, \operatorname{Pic}(C)=\operatorname{Div}(C) / \operatorname{Div}_{P}(C)$ the Picard group, and $\operatorname{Pic}_{0}(C)=\operatorname{Div}_{0}(C) / \operatorname{Div}_{P}(C)$. Given a finite or cofinite set of places $T$ of $K$, we write $\operatorname{Div}(T)$ for the subgroup of divisors whose support is contained in $T$. Similarly we introduce the subgroups $\operatorname{Div}_{0}(T)$ and $\operatorname{Div}_{P}(T)$.

Let $S \subseteq C$ be a finite set of places of $K$ such that $\operatorname{Div}(S)$ represents all classes in $\operatorname{Pic}(C)$. (For example, if $C=\mathbb{P}^{1}$ then $S$ may be any set containing a place of degree 1.) Let $F=\sum_{v \in S} n_{v} v$ be an effective divisor, with $n_{v} \geqslant 1$ for all $v \in S$. The divisor $F$ will play the role of conductor. Let $\operatorname{Div}_{P, F}(C-S)$ be the subgroup of principal divisors $(f)$ with $f \in K^{\times}$congruent to 1 modulo $F$; that is, $\operatorname{ord}_{v}(f-1) \geqslant n_{v}$ for all places $v \in S$. Let $\operatorname{Pic}_{F}(C)=\operatorname{Div}(C-S) / \operatorname{Div}_{P, F}(C-S)$ be the ray class group modulo $F$ and $\operatorname{Pic}_{F, 0}(C)=\operatorname{Div}_{0}(C-S) / \operatorname{Div}_{P, F}(C-S)$ be the degree 0 subgroup. Choose a divisor $B_{1} \in \operatorname{Div}(C-S)$ of degree 1 and a positive integer $n$. Then we define

$$
X(F, n)=\operatorname{Div}(C-S) /\left\langle n B_{1}\right\rangle+\operatorname{Div}_{P, F}(C-S) .
$$

This is a finite group. Let $\rho$ be a character of $X(F, n)$. Then in an obvious way $\rho$ corresponds to a pair: a character on $\operatorname{Pic}_{F, 0}(C)$ and a value $\rho\left(B_{1}\right)=\omega$, where $\omega$ is an $n$th root of unity. Since $\rho$ is trivial on $\operatorname{Div}_{P, F}(C-S)$ one says that $\rho$ has conductor $F$; the exact conductor $F_{\rho}$ is by definition the smallest divisor $F_{\rho} \leqslant F$ such that $\rho$ is trivial on $\operatorname{Div}_{P, F_{\rho}}(C-S)$. If $F_{\rho}=F$ then $\rho$ is primitive.

Let $T$ be a finite set of places of $C$; almost always, we will take $T \supseteq S$. Let $\rho$ be an automorphic character of $\operatorname{Div}(C-T)$, so that the conductor $F_{\rho}$ is supported on $T$. We define the $L$-series

$$
L(s, \rho ; C-T)=\prod_{v \notin T}\left(1-\rho(v)|v|^{-s}\right)^{-1},
$$




\section{Double Dirichlet SERIES OVER FUNCTION FIELDS}

where $|v|$ is the norm of the prime corresponding to $v$. Extend $|\cdot|$ multiplicatively to effective divisors. Then the Euler product is also a sum over effective divisors:

$$
L(s, \rho ; C-T)=\sum_{0 \leqslant D \in \operatorname{Div}(C-T)} \rho(D)|D|^{-s} .
$$

The above expressions are valid for $\operatorname{Re}(s)>1$. By the theory of $\operatorname{GL}(1) L$-functions, these series have analytic continuation and functional equation; see for example [Wei74] or [Mor91]. (Note that our partial $L$-function $L(s, \rho ; C-T)$ differs from the complete $L$-function by at most a finite number of Euler factors.) In particular, if $\rho$ is not trivial on $\operatorname{Div}_{0}(C-T)$ then $L(s, \rho ; C-T)$ is entire [Wei74, ch. VII, Theorem 6], while if $\rho$ is trivial on $\operatorname{Div}_{0}(C-T)$ and $\rho\left(B_{1}\right)=\omega\left(\right.$ so $\left.\rho(D)=\omega^{\operatorname{deg}(D)}\right)$ then $\left(1-\omega q^{1-s}\right) L(s, \rho ; C-T)$ is entire. (See [Wei74, ch. VII, Theorem 4]. Note that in this situation $L(s, \rho ; C-T)=\zeta_{K, T}\left(s-\log _{q}(\omega)\right)$, where $\zeta_{K, T}$ denotes the zeta-function with the Euler factors at places in $T$ removed.) Also, if $\rho$ is a character of $X(F, n)$, and $\chi$ is any Hecke character, whose conductor has support $S_{\chi}$, we shall form the twisted $L$-function $L\left(s, \rho \chi ; C-S-S_{\chi}\right)$. We do not assume that the exact conductor of $\rho \chi$ has support $S \cup S_{\chi}$; it may be smaller.

In this paper we wish to consider a sum of such twisted $L$-series, the twists given by quadratic characters. These characters correspond naturally to classes of $K^{\times} /\left(K^{\times}\right)^{2}$. It is well known how to write such a sum in the case of trivial Picard group $\operatorname{Pic}(C) \cong \mathbb{Z}$ ('class number $1^{\prime}$ ); compare [HR92]. To do so in the case of non-trivial Picard group, we first choose certain representatives essentially parametrizing the divisor classes modulo squares; then the remaining divisor classes are parametrized by a similar set of field elements. Alternatively, one may describe the construction below as allowing twisting by the (not-necessarily-quadratic) characters obtained by extending the natural quadratic character on principal divisors up to the full group of divisors, then summing these objects, the extensions chosen compatibly so that the sum satisfies functional equations: see Remark 1.3 below. Though the divisor class representatives and extensions are not canonical, the vector space of twisted sums is finite-dimensional, and is canonical. We proceed as follows.

For $m \in K^{\times}$and $D \in \operatorname{Div}(C)$, let $\left(\frac{m}{D}\right)$ be the usual quadratic residue symbol

$$
\left(\frac{m}{D}\right)=\prod_{v, \operatorname{ord}_{v}(m)=0}\left(\frac{m}{v}\right)^{\operatorname{ord}_{v}(D)},
$$

where the right-hand side is a product of standard Legendre symbols (cf. [CF67, Exercise 1]). Let $\chi_{m}$ denote the character associated to $K(\sqrt{m}) / K$ by class field theory: $\chi_{m}(D)=\left(\frac{m}{D}\right)$ if $D$ is disjoint from $(m)$, and $\chi_{m}((n))=1$ if $n \equiv 1$ modulo the square-free part of $(m)$. Choose a set $\mathcal{E} \subseteq \operatorname{Div}_{\geqslant 0}(C-S)$ of coset representatives for the quotient

$$
\operatorname{Div}(C-S) / 2 \operatorname{Div}(C-S)+\operatorname{Div}_{P, F}(C-S) \cong \operatorname{Pic}_{F}(C) \otimes \mathbb{Z} / 2 \mathbb{Z} .
$$

(We shall simply identify these two groups from now on.) Also, for each $E \in \mathcal{E}$ choose $m_{E}$ so that $E-\left(m_{E}\right) \in \operatorname{Div}(S)$.

Let $D=E+(m)+2 G \in \operatorname{Div}(C-S)$, with $E \in \mathcal{E}, G \in \operatorname{Div}(C-S)$ and $m \equiv 1(\bmod F)$. Let $m_{D}=m m_{E}$ and define

$$
\chi_{D}=\chi_{m_{D}}
$$

(For convenience, we assume that $0 \in \mathcal{E}$ and that $m_{0}=1$, so that $\chi_{(m)}$ is the same as $\chi_{m}$ if $m \equiv 1$ $(\bmod F)$.) Then $\chi_{D}$ is well defined, according to the following lemma.

Lemma 1.1. Let $D \in \operatorname{Div}(C-S)$. Write

$$
D=E+(m)+2 G
$$

with $E \in \mathcal{E}, G \in \operatorname{Div}(C-S)$, and $m \equiv 1(\bmod F)$. If $D=E^{\prime}+\left(m^{\prime}\right)+2 G^{\prime}$ is another such expression then $E^{\prime}=E$ and $m^{\prime} / m \in\left(K^{\times}\right)^{2}$. 


\section{B. Fisher AND S. FriedberG}

Proof. Let $E \in \mathcal{E}$ be the coset representative corresponding to $D$. Then we may write $D=E+$ $(m)+2 G$ as above. If $D=E^{\prime}+\left(m^{\prime}\right)+2 G^{\prime}$, then since $\mathcal{E}$ is a set of coset representatives, $E=E^{\prime}$. Let $n=m^{\prime} / m$ and let $S_{1}$ denote the support of the divisor $G-G^{\prime}$, so that $(n)=2\left(G-G^{\prime}\right) \in$ $\operatorname{Div}_{P, F}(C-S) \cap 2 \operatorname{Div}\left(S_{1}\right)$. Then $\chi_{n}(H)=1$ for all $H \in \operatorname{Div}(S)$ since $n \equiv 1(\bmod F)$, while $\chi_{n}\left(\left(n^{\prime}\right)\right)=\chi_{(n)}\left(\left(n^{\prime}\right)\right)=1$ by quadratic reciprocity for all $\left(n^{\prime}\right) \in \operatorname{Div}_{P}\left(C-S_{1}\right)$ since $(n) \in 2 \operatorname{Div}\left(S_{1}\right)$. It follows that by quadratic reciprocity $\chi_{n}(H)=1$ for all $H \in \operatorname{Div}(S)+\operatorname{Div}_{P}\left(C-S_{1}\right)=\operatorname{Div}\left(C-S_{1}\right)$, whence $n$ is a square by class field theory.

Note that $\chi_{D}$ depends on the choice of $m_{E}$ but not on the choice of $m$, thanks to Lemma 1.1. Also, if $D_{0} \in \operatorname{Div}(C-S)$, then $\chi_{D+2 D_{0}}=\chi_{D}$. It is these quadratic characters that we shall sum in the double Dirichlet series.

Next let us define the correction factor, which must multiply the $L$-functions in the double sum. Fix $F$ and $n$ as above (these will play the role of conductor), and let $\rho$ be a character of $X(F, n)$. Let $\mu$ be the Möbius function on $\operatorname{Div}(C)$. Let $0 \leqslant D \in \operatorname{Div}(C-S)$, and let $S_{D}$ denote the support of the conductor of $\chi_{D}$. Define the finite sum

$$
a(s, \rho, D)=\sum_{\substack{0 \leqslant d_{1} \in \operatorname{Div}\left(C-S-S_{D}\right) \\ 0 \leqslant d_{2} \in \operatorname{Div}(C-S) \\ 2\left(d_{1}+d_{2}\right) \leqslant D}} \mu\left(d_{1}\right) \chi_{D}\left(d_{1}\right) \rho\left(d_{1}+2 d_{2}\right)\left|d_{1}\right|^{-s}\left|d_{2}\right|^{1-2 s} .
$$

In the rational function field case, this function arises in the Whittaker expansion of the metaplectic Eisenstein series. Also define

$$
L(s, \rho, D)=L\left(s, \rho \chi_{D} ; C-S-S_{D}\right) a(s, \rho, D) .
$$

Finally, we define the double Dirichlet series of concern to us in this paper. Let $\rho_{1}$ and $\rho_{2}$ be characters of $X(F, n)$. Define

$$
Z\left(s, w ; \rho_{1}, \rho_{2}\right)=\sum_{0 \leqslant D \in \operatorname{Div}(C-S)} \rho_{2}(D) L\left(s, \rho_{1}, D\right)|D|^{-w} .
$$

It is not difficult to check that the series converges absolutely in the tube domain $\operatorname{Re}(s)>1$, $\operatorname{Re}(w)>1$. Note that the definition of the characters $\chi_{D}$ depends on the choice of the representatives $\mathcal{E} \subseteq \operatorname{Div}(C-S)$, and the field elements $m_{E}, E \in \mathcal{E}$. Accordingly, this sum of $L$-functions also depends on these choices. The definition also makes use of a degree 1 divisor $B_{1} \in \operatorname{Div}(C-S)$. However, varying over characters $\rho_{1}, \rho_{2}$ gives a finite-dimensional vector space, which depends only on $F$ and $n$. That is, the following proposition holds.

Proposition 1.2. Let $n$ be even and let $V(F, n)$ be the span of $Z\left(s, w ; \rho_{1}, \rho_{2}\right)$ for $\rho_{1}, \rho_{2} \in \hat{X}(F, n)$. Then $V(F, n)$ is a finite-dimensional vector space of functions, which is independent of the choices of the representatives $\mathcal{E}$ and the field elements $m_{E}, E \in \mathcal{E}$. If in addition the exponent of the group $\operatorname{Pic}_{F, 0}(C)$ divides $n$ then the vector space is independent of the choice of the degree 1 divisor $B_{1}$.

We give the proof of this proposition in the next paragraph, but first it is convenient to extend the notation to linear combinations of characters. That is, let $R(F, n)$ be the vector space of functions on $X(F, n)$ with values in $\mathbb{C}$. Since $X(F, n)$ is a finite abelian group, its characters form a basis of $R(F, n)$. If $\sigma_{j} \in R(F, n), j=1,2$ and $\sigma_{j}=\sum c_{i, j} \rho_{i}$ with the $\rho_{i}$ characters of $X(F, n)$ and $c_{i, j} \in \mathbb{C}$, then define

$$
Z\left(s, w ; \sigma_{1}, \sigma_{2}\right)=\sum_{i_{1}, i_{2}} c_{i_{1}, 1} c_{i_{2}, 2} Z\left(s, w ; \rho_{i_{1}}, \rho_{i_{2}}\right) .
$$

In particular, for $x \in X(F, n)$, let $\delta_{x}$ denote the function on $\operatorname{Div}(C-S)$ given by $\delta_{x}(D)=1$ if the class of $D$ in $X(F, n)$ is $x, \delta_{x}(D)=0$ otherwise. Then the $\delta_{x}$, for $x \in X(F, n)$, give another basis of 


\section{DOUble DiRICHLET SERIES OVER FUNCTION FIELDS}

$R(F, n)$, and $Z\left(s, w ; \rho, \delta_{x}\right)$ is a partial Dirichlet series, the sum over all effective divisors of the form $D=E+(m)+n j B_{1}$ with $m \equiv 1(\bmod F)$ and $j$ an integer, where $E$ is a fixed representative of $x$.

Proof of Proposition 1.2. Let $n$ be even. Then the vector space $V(F, n)$ is spanned by the partial sums

$$
Z\left(s, w ; \rho, \delta_{x}\right)=\sum L(s, \rho, D)|D|^{-w},
$$

$x \in X(F, n)$, where the sum is over $D$ as in (1.4) with the additional condition that $D$ projects to $x$ in $X(F, n)$. Let us consider the effect of changing the choices on these sums. Since $n$ is even there is a natural projection from $X(F, n)$ to $\operatorname{Pic}_{F}(C) \otimes \mathbb{Z} / 2 \mathbb{Z}$. Let $E \in \mathcal{E}$ be the representative for the class of $x$. First, suppose that $E-\left(m_{E}^{\prime}\right) \in \operatorname{Div}(S)$ and one chooses $m_{E}^{\prime}$ in place of $m_{E}$ in this construction. In view of (1.1), (1.2), and (1.3), it follows that each character $\chi_{D}$ in the sum (1.6) is replaced by $\chi_{D} \chi_{m_{E}^{\prime} / m_{E}}$. Thus with this choice, the sum (1.6) is replaced by $Z\left(s, w ; \rho \chi_{m_{E}^{\prime} / m_{E}}, \delta_{x}\right)$. Since $\left(m_{E}^{\prime} / m_{E}\right) \in \operatorname{Div}(S)$, the character $\chi_{m_{E}^{\prime} / m_{E}} \in \hat{X}(F, 2)$. Thus the span of the new sums is identical to the span of the original ones.

Next we turn to the choice of representatives. Suppose that $D, E, E^{\prime}$ are in the same class in $\operatorname{Pic}_{F}(C) \otimes \mathbb{Z} / 2 \mathbb{Z}$. Let us compare the character $\chi_{D}$ obtained by using $E$ as a representative to the character obtained using $E^{\prime}$. Write $E^{\prime}=E+\left(m^{\prime}\right)+2 G^{\prime}$ as in Lemma 1.1, and further write $G^{\prime}=G_{0}+\left(m_{0}\right)$ with $G_{0} \in \operatorname{Div}(S), m_{0} \in K^{\times}$. (Here we use that $\operatorname{Div}(S)$ maps onto $\operatorname{Pic}(C)$.) Then $E^{\prime}-\left(m_{E} m^{\prime} m_{0}^{2}\right)=E-\left(m_{E}\right)+2 G_{0} \in \operatorname{Div}(S)$. So we may choose $m_{E^{\prime}}=m_{E} m^{\prime} m_{0}^{2}$ (cf. the paragraph above). Further, if $D=E^{\prime}+(m)+2 G$ as in Lemma 1.1, then $D=E+\left(m m^{\prime}\right)+2\left(G+G^{\prime}\right)$. Thus the character $\chi_{D}$ using $E$ as representative gives $\chi_{m m^{\prime} m_{E}}$ while using $E^{\prime}$ as representative we obtain $\chi_{m m_{E^{\prime}}}=\chi_{m m_{E} m^{\prime} m_{0}^{2}}$. These are equal, and the independence follows.

Finally, suppose that $n$ is divisible by the exponent of $\operatorname{Pic}_{F, 0}(C)$. If $B_{1}^{\prime}$ is another degree 1 divisor, then $B_{1}^{\prime}-B_{1} \in \operatorname{Div}_{0}(C-S)$. Under this divisibility hypothesis, $n\left(B_{1}^{\prime}-B_{1}\right)$ projects to the trivial class in $\operatorname{Pic}_{F}(C)$. It follows that $\left\langle n B_{1}\right\rangle+\operatorname{Div}_{P, F}(C-S)=\left\langle n B_{1}^{\prime}\right\rangle+\operatorname{Div}_{P, F}(C-S)$. Thus $X(F, n)$ is independent of the choice of $B_{1}$. (See also Remark 1.5 below.)

Remark 1.3. Associating quadratic characters $\chi_{D}$ to divisors $D$ in this way is in fact equivalent to choosing a compatible system of extensions of quadratic characters defined on principal divisors to the full divisor group. This allows one to give a canonical definition of the vector space $V(F, n)$, as follows.

Let $D \in \operatorname{Div}(C-S)$. For this remark only, let $S_{D}$ denote the support of the square-free part of $D$. Then the standard quadratic symbol gives a character of $\operatorname{Div}_{P, F}\left(C-S-S_{D}\right)$ which we shall denote $\lambda_{D}$. That is, if $m \in K^{\times}, m \equiv 1 \bmod F$, then define $\lambda_{D}((m))=\left(\frac{m}{D}\right)$. It is not difficult to check that $\lambda_{D}$ is the restriction of $\chi_{D}$ to $\operatorname{Div}_{P, F}\left(C-S-S_{D}\right)$. For each effective divisor $D \in \operatorname{Div}(C-S)$ let $\tilde{\lambda}_{D}$ denote a Hecke character whose restriction to $\operatorname{Div}_{P, F}\left(C-S-S_{D}\right)$ matches $\lambda_{D}$. (Note that the induced space $\operatorname{Ind}_{\operatorname{Div}_{P, F}\left(C-S-S_{D}\right)}^{\operatorname{Div}_{D}\left(C-S-S_{D}\right)} \lambda_{D}$ naturally decomposes into a direct sum of characters each of which extends the character $\lambda_{D}$; the Hecke characters $\tilde{\lambda}_{D}$, restricted to the degree 0 divisors, are precisely the characters in this induced space.) Let us say that a family of extensions $\tilde{\lambda}=\left\{\tilde{\lambda}_{D}\right\}, D$ ranging over the effective divisors in $\operatorname{Div}(C-S)$, is compatible if, whenever $D=D^{\prime}+(m)+2 G$ with $m \equiv 1 \bmod F$, then the extensions $\tilde{\lambda}_{D}, \tilde{\lambda}_{D^{\prime}}$ satisfy

$$
\tilde{\lambda}_{D}=\tilde{\lambda}_{D^{\prime}} \chi_{m}
$$

on $\operatorname{Div}\left(C-S-S_{D}-S_{D^{\prime}}\right)$. Then the space $V(F, n)$ may also be described as the span of the double Dirichlet series

$$
\sum_{0 \leqslant D \in \operatorname{Div}(C-S)} \rho_{2}(D) L\left(s, \rho_{1} \tilde{\lambda}_{D} ; C-S-S_{D}\right) a\left(s, \rho_{1} \tilde{\lambda}_{D}, D\right)|D|^{-w}
$$




\section{B. Fisher AND S. FriedberG}

formed from all compatible families of extensions $\tilde{\lambda}$ and all $\rho_{1}, \rho_{2} \in \hat{X}(F, n)$. This space is also obtained from any fixed $\tilde{\lambda}$ and all $\rho_{1}, \rho_{2} \in \hat{X}(F, n)$.

Remark 1.4. It is possible to make choices in such a way that $\chi_{D+D^{\prime}}=\chi_{D} \cdot \chi_{D^{\prime}}$ for all $D, D^{\prime} \in$ $\operatorname{Div}(C-S)$. To do this, choose a basis $\left\{e_{1}, \ldots, e_{k}\right\}$ of $\operatorname{Pic}_{F}(C) \otimes \mathbb{Z} / 2 \mathbb{Z}$. For each $j$, choose an effective divisor $E_{j} \in \operatorname{Div}(C-S)$ representing $e_{j}$, and choose $m_{j}=m_{E_{j}}$ so that $E_{j}-\left(m_{j}\right) \in \operatorname{Div}(S)$. Let $\mathcal{E}$ be the set of all $E=\sum_{j} c_{j} E_{j}$ with $c_{j} \in\{0,1\}$. For each such $E$, choose $m_{E}=\prod_{j} m_{j} c_{j}$. With these choices, $\chi_{E+E^{\prime}}=\chi_{E} \cdot \chi_{E^{\prime}}$ whenever $E, E^{\prime} \in \mathcal{E}$, which implies the claim.

Remark 1.5. Let $\sigma$ be a character of $\operatorname{Pic}_{F, 0}(C)$. For any $n$th root of unity $\omega$, we extend $\sigma$ to a character $\sigma_{(\omega)}$ of $X(F, n)$ by declaring $\sigma_{(\omega)}\left(B_{1}\right)=\omega$. For any $D \in \operatorname{Div}(C-S)$ of degree $d$, we then have $\sigma_{(\omega)}(D)=\sigma\left(D-d B_{1}\right) \cdot \omega^{d}$. Next, for $j \in \mathbb{Z} / n \mathbb{Z}$, define $\sigma_{[j]}=(1 / n) \sum_{\omega^{n}=1} \omega^{-j} \sigma_{(\omega)}$, so that $\sigma_{[j]}(D)=\sigma\left(D-d B_{1}\right)$ if $d \equiv j(\bmod n)$, and $\sigma_{[j]}(D)=0$ if $d \not \equiv j(\bmod n)$. We can also express $\sigma_{(\omega)}$ as $\sum_{j} \omega^{j} \sigma_{[j]}$. Thus $Z\left(s, w ; \rho, \sigma_{[j]}\right)$ is exactly the sum of the terms in $Z\left(s, w ; \rho, \sigma_{(1)}\right)$ involving $q^{-a s-b w}$ with $b \equiv j(\bmod n)$. Theorem 3.3 implies a similar interpretation of $Z\left(s, w ; \sigma_{[j]}, \rho\right)$. In particular, the series of the form $Z\left(s, w ; \sigma_{(1)}, \tau_{(1)}\right)$ determine all of the $Z\left(s, w ; \rho_{1}, \rho_{2}\right)$.

Suppose now that $n \cdot \operatorname{Pic}_{F, 0}(C)=0$, and let $B_{1}^{\prime}$ be another divisor of degree 1 . If we extend $\sigma$ to $\sigma_{(\omega)}^{\prime}$ by defining $\sigma_{(\omega)}^{\prime}\left(B_{1}^{\prime}\right)=\omega$, then $\sigma_{(\omega)}^{\prime}(D)=\sigma\left(B_{1}-B_{1}^{\prime}\right)^{\operatorname{deg}(D)} \cdot \sigma_{(\omega)}(D)$, whence $\sigma_{[j]}^{\prime}=$ $\sigma\left(B_{1}-B_{1}^{\prime}\right)^{j} \cdot \sigma_{[j]}$. Thus, except for the constant factor $\sigma\left(B_{1}-B_{1}^{\prime}\right)^{j}, Z\left(s, w ; \rho, \sigma_{[j]}\right)$ is independent of the choice of $B_{1}$.

\section{The first functional equation}

The goal of this section is to derive a functional equation for the double Dirichlet series from that of the $L$-functions $L\left(s, \rho_{1} \chi_{D} ; C-S-S_{D}\right)$ that are summed in $Z(s, w)$. This functional equation describes the action of an element of $V(F, n)$ under the involution $(s, w) \rightarrow(1-s, w+s-1 / 2)$. The main ingredient here is a detailed study of the epsilon-factor for $L\left(s, \rho_{1}\right)$ under twisting by a quadratic character. We begin by recalling the functional equation, especially the definition of the epsilon-factor. A convenient reference is [Mor91, Theorem 3.4].

Let $\rho$ be a character of exact conductor $F_{\rho}=\sum_{v} e_{v} v$. Then the functional equation is given by

$$
L\left(s, \rho ; C-F_{\rho}\right)=\epsilon(\rho)\left|(\Omega)+F_{\rho}\right|^{1 / 2-s} L\left(1-s, \rho^{-1} ; C-F_{\rho}\right),
$$

where $(\Omega)$ is a canonical divisor, so that $|(\Omega)|=q^{2 g-2}$, where $g$ is the genus of $K$, and $\epsilon(\rho)$ has absolute value 1 . The epsilon-factor may be computed as follows. Let $\Omega$ be a meromorphic differential on $C$ such that $(\Omega)+F_{\rho}$ is disjoint from $F_{\rho}$ and let $e: \mathbb{F}_{q} \rightarrow \mathbb{C}^{\times}$be the standard additive character. Define normalized local and global Gauss sums by

$$
G_{v}(\rho, \Omega)=q^{-e_{v} \operatorname{deg}(v) / 2} \sum_{x \in\left(\mathcal{O}_{v} /\left(\pi_{v} e_{v}\right)\right)^{\times}} \bar{\rho}_{v}(x) e\left(\operatorname{Res}_{v}(x \Omega)\right)
$$

and

$$
G(\rho, \Omega)=\prod_{v} G_{v}(\rho, \Omega)
$$

where the product is over $v \in \operatorname{Supp}\left(F_{\rho}\right)$. Then

$$
\epsilon(\rho)=\rho\left((\Omega)+F_{\rho}\right) / G(\rho, \Omega) .
$$

(In [Mor91], conditions are imposed on $\Omega$ that determine not only the epsilon-factor but also its numerator and denominator.)

Remark 2.1. If $m \in K^{\times}$, then the quadratic character $\chi_{m}$ has epsilon-factor $\epsilon\left(\chi_{m}\right)=1$. This property follows from $(2.2)$, but is easier to see from the functional equation: we can express $L\left(s, \chi_{m}\right)$ 


\section{Double Dirichlet SERIES OVER FUNCTION FIELDS}

as a ratio of zeta-functions, each of which has a functional equation with $\epsilon=1$. Note also that the Gauss sum $G(\rho)$ depends only on $\rho$ restricted to principal divisors.

We shall apply the following lemma only in the case that $\sigma$ and $\tau$ are quadratic characters, but it is just as easy to prove this more general version.

Lemma 2.2. Let $\rho, \sigma$, and $\tau$ be characters of $\operatorname{Div}(C)$ such that the conductors $F_{\sigma / \tau}$ and $F_{\rho}$ have disjoint supports. Then

$$
\epsilon(\rho \sigma)=\epsilon(\rho \tau) \cdot \epsilon(\sigma) / \epsilon(\tau) \cdot \rho\left(F_{\sigma}-F_{\tau}\right) \cdot(\sigma / \tau)\left(F_{\rho \sigma}-F_{\sigma}\right) .
$$

Proof. Let $T=\operatorname{Supp}\left(F_{\rho}+F_{\sigma}+F_{\tau}\right)$. In order to compute the epsilon-factors, first choose a differential $\Omega$ and choose $\xi, \eta \in K^{\times}$with $\xi \equiv 1\left(\bmod F_{\rho}\right)$ and $\eta \equiv 1\left(\bmod F_{\sigma / \tau}\right)$ such that

$$
(\Omega)+F_{\rho \tau}, \quad(\xi)+F_{\sigma}-F_{\tau},(\eta)+F_{\tau}-F_{\rho \tau} \in \operatorname{Div}(C-T) .
$$

As the hypothesis of disjoint supports implies that $F_{\rho \sigma}-F_{\rho \tau}=F_{\sigma}-F_{\tau}$, we may use $\Omega, \xi \Omega, \eta \Omega$, and $\xi \eta \Omega$ to compute the epsilon-factors for $\rho \tau, \rho \sigma, \tau$, and $\sigma$, respectively.

We can now calculate the ratio of epsilon-factors

$$
\frac{\epsilon(\rho \sigma) \epsilon(\tau)}{\epsilon(\rho \tau) \epsilon(\sigma)}=\frac{G(\rho \tau, \Omega) G(\sigma, \xi \eta \Omega)}{G(\rho \sigma, \xi \Omega) G(\tau, \eta \Omega)} \cdot \frac{(\rho \sigma)\left((\xi \Omega)+F_{\rho \sigma}\right) \tau\left((\eta \Omega)+F_{\tau}\right)}{(\rho \tau)\left((\Omega)+F_{\rho \tau}\right) \sigma\left((\xi \eta \Omega)+F_{\sigma}\right)} .
$$

Writing the Gauss sums as products of local terms, the first fraction on the right-hand side of (2.3) is 1. Since $F_{\rho \sigma}-F_{\rho \tau}=F_{\sigma}-F_{\tau}$, we can rewrite $(2.3)$ as

$$
\frac{\epsilon(\rho \sigma) \epsilon(\tau)}{\epsilon(\rho \tau) \epsilon(\sigma)}=\rho\left((\xi)+F_{\sigma}-F_{\tau}\right) \cdot(\sigma / \tau)\left(-(\eta)+F_{\rho \sigma}-F_{\sigma}\right) .
$$

The conditions on $\xi$ and $\eta$ imply that $\rho((\xi))=(\sigma / \tau)((\eta))=1$, so the lemma now follows from (2.4).

Corollary 2.3. Let $\rho$ be a character of $\operatorname{Div}(C)$ and let $m, n \in K^{\times}$such that $m / n \equiv 1\left(\bmod F_{\rho}\right)$. Then $\epsilon\left(\rho \chi_{m}\right)=\epsilon\left(\rho \chi_{n}\right) \cdot \rho\left(F_{m}-F_{n}\right)$, where $F_{m}$ and $F_{n}$ denote the conductors of $\chi_{m}$ and $\chi_{n}$, respectively.

Proof. This follows from Remark 2.1 and Lemma 2.2, taking $\sigma=\chi_{m}$ and $\tau=\chi_{n}$. The hypothesis $m / n \equiv 1\left(\bmod F_{\rho}\right)$ implies both that $F_{\sigma / \tau}$ is disjoint from $F_{\rho}$ and that $(\sigma / \tau)\left(F_{\rho \sigma}-F_{\sigma}\right)=1$.

Lemma 2.4. Let $\rho$ be a character of $X(F, n)$ and let $D=D_{0}+2 D_{1} \in \operatorname{Div}(C-S)$, with $D_{0}, D_{1} \geqslant 0$ and $D_{0}$ square-free. Let $F_{\rho, D}$ denote the conductor of $\rho \chi_{D}$ and $S_{\rho, D}$ denote the support of $F_{\rho, D}$. Then

$$
\frac{L(s, \rho, D)}{L\left(1-s, \rho^{-1}, D\right)}=\epsilon\left(\rho \chi_{D}\right) \rho\left(2 D_{1}\right)\left|(\Omega)+F_{\rho, D}+2 D_{1}\right|^{1 / 2-s} \prod_{v} \frac{1-\rho \chi_{D}(v)|v|^{-s}}{1-\rho^{-1} \chi_{D}(v)|v|^{s-1}},
$$

where the product is over $v \in S-S_{\rho, D}$, and $(\Omega)$ is a canonical divisor.

Note that $\left|(\Omega)+F+2 D_{1}\right|=q^{2 g-2+\operatorname{deg}\left(F+2 D_{1}\right)}$, where $F=F_{\rho, D}$ and $g$ is the genus of $K$.

Proof. Letting $S_{D}$ denote the support of $\chi_{D},(1.3)$ gives

$$
\begin{aligned}
L(s, \rho, D) & =L\left(s, \rho \chi_{D} ; C-S-S_{D}\right) a(s, \rho, D) \\
& =L\left(s, \rho \chi_{D} ; C-S_{\rho, D}\right) a(s, \rho, D) \prod_{v}\left(1-\rho \chi_{D}(v)|v|^{-s}\right),
\end{aligned}
$$




\section{B. Fisher AND S. FriedBerG}

with the product as described above. There is a simple functional equation for the correction factors. Indeed,

$$
\begin{aligned}
a(s, \rho, D) & =\sum_{\substack{d_{1}, d_{2}, d_{3} \geqslant 0 \\
d_{1} \in \operatorname{Div}\left(C-S-S_{D}\right) \\
d_{1}+d_{2}+d_{3}=D_{1}}} \mu\left(d_{1}\right) \chi_{D}\left(d_{1}\right) \rho\left(d_{1}+2 d_{2}\right)\left|d_{1}\right|^{-s}\left|d_{2}\right|^{1-2 s} \\
& =\rho\left(2 D_{1}\right)\left|D_{1}\right|^{1-2 s} \sum_{d_{1}, d_{2}, d_{3}} \mu\left(d_{1}\right) \chi_{D}\left(d_{1}\right) \rho^{-1}\left(d_{1}+2 d_{3}\right)\left|d_{1}\right|^{s-1}\left|d_{3}\right|^{2 s-1} .
\end{aligned}
$$

Thus

$$
a(s, \rho, D)=\rho\left(2 D_{1}\right)\left|D_{1}\right|^{1-2 s} a\left(1-s, \rho^{-1}, D\right) .
$$

Combining this with the functional equation (2.1) for $L\left(s, \rho \chi_{D} ; C-S_{\rho, D}\right)$ gives the result.

Corollary 2.5. Let $n$ be even and let $\rho_{1}, \rho_{2}$ be characters of $X(F, n)$. Let

$$
\phi\left(s, \rho_{1}\right)= \begin{cases}1-\rho_{1}^{2}\left(B_{1}\right) q^{2-2 s} & \text { if } \rho_{1}^{2}=1 \text { on } \operatorname{Div}_{0}(C-S), \\ 1 & \text { otherwise. }\end{cases}
$$

Then the series for $\phi\left(s, \rho_{1}\right) Z\left(s, w ; \rho_{1}, \rho_{2}\right)$ given via (1.4) converges absolutely and uniformly on compacta and is uniformly bounded away from the boundary of the tube domain described by the condition $\operatorname{Re}(w)>\max \left\{1, \frac{3}{2}-\operatorname{Re}(s), \frac{3}{2}-\frac{1}{2} \operatorname{Re}(s)\right\}$.

Proof. Let $s=\sigma+i t$. For $\operatorname{Re}(s)>1$, it is immediate that $\left|L\left(s, \rho_{1} \chi_{D} ; C-S-S_{D}\right)\right| \leqslant \zeta_{K, S}(\sigma)$. From (1.2) and (1.3) it follows that $\left|L\left(s, \rho_{1}, D\right)\right|=\mathrm{O}\left(\zeta_{K, S}(\sigma)|D|^{\delta}\right)$ for every $\delta>0$, the implied constant depending only on $K$, and the statements of the corollary follow. Suppose next that $\operatorname{Re}(s)<0$. Then the functional equation given by Lemma 2.4 implies that, for all $\delta>0$,

$$
\left|L\left(s, \rho_{1}, D\right)\right|=\mathrm{O}\left(F(\sigma) \zeta_{K, S}(1-\sigma)|D|^{1 / 2+\delta-\sigma}\right),
$$

where $F(\sigma)$ is a power of $q^{1-\sigma}$ depending on the genus $g$ and the primes in $S$. Once again the implied constant depends only on $K$. This estimate implies the result for $\operatorname{Re}(s)<0, \operatorname{Re}(s+w-1 / 2)>1$. Since $\phi\left(s, \rho_{1}\right) L\left(s, \rho_{1}, D\right)$ is always holomorphic, applying standard convexity methods one may fill in the region $0 \leqslant \operatorname{Re}(s) \leqslant 1$. The corollary follows.

Theorem 2.6. Assume that $n$ is even, and let $x \in X(F, n)$. Let $\delta_{x}$ denote the corresponding characteristic function on $\operatorname{Div}(C-S): \delta_{x}(D)=1$ if $x$ is the class of $D, \delta_{x}(D)=0$ otherwise. Let $E \in \mathcal{E}$ represent the class of $x$ in $X(F, n) \otimes \mathbb{Z} / 2 \mathbb{Z}=\operatorname{Pic}_{F}(C) \otimes \mathbb{Z} / 2 \mathbb{Z}$. Then for any character $\rho$ of $X(F, n)$

$$
Z\left(s, w ; \rho, \delta_{x}\right)=\frac{L(s, \rho, E)}{L\left(1-s, \rho^{-1}, E\right)} \cdot \rho(x-E)|E|^{s-1 / 2} Z\left(1-s, s+w-\frac{1}{2} ; \rho^{-1}, \delta_{x}\right)
$$

on the tube domain described by the condition $\operatorname{Re}(w)>\max \left\{1, \frac{3}{2}-\operatorname{Re}(s), \frac{3}{2}-\frac{1}{2} \operatorname{Re}(s)\right\}$.

Proof. From the definitions (1.4) and (1.5), $Z\left(s, w ; \rho, \delta_{x}\right)=\sum_{D} \delta_{x}(D) L(s, \rho, D)|D|^{-w}$, where the sum is over $0 \leqslant D \in \operatorname{Div}(C-S)$. By Corollary 2.5, $\phi(s, \rho)$ times this series converges in the domain we are considering. Assume that $\delta_{x}(D)=1$, so that $D$ contributes to the sum. Then we can write $D=E+(m)+2 G$ as in Lemma 1.1, with $E \in \mathcal{E}$ as above. In particular, $\rho \chi_{D}(v)=\rho \chi_{E}(v)$ for any $v \in S-S_{\rho, D}=S-S_{\rho, E}$. Applying Lemma 2.4 to $D$ and to $E$, we obtain

$$
\frac{L(s, \rho, D) / L\left(1-s, \rho^{-1}, D\right)}{L(s, \rho, E) / L\left(1-s, \rho^{-1}, E\right)}=\frac{\epsilon\left(\rho \chi_{D}\right)}{\epsilon\left(\rho \chi_{E}\right)} \cdot \rho\left(2 D_{1}-2 E_{1}\right) \cdot\left|F_{\rho, D}-F_{\rho, E}+2 D_{1}-2 E_{1}\right|^{1 / 2-s},
$$

where $D=D_{0}+2 D_{1}$ and $E=E_{0}+2 E_{1}$, with $D_{0}$ and $E_{0}$ square-free. According to Corollary 2.3, the ratio of epsilon-factors gives $\rho\left(F_{D}-F_{E}\right)$, where $F_{D}$ and $F_{E}$ denote the conductors of $\chi_{D}$ and $\chi_{E}$, 
respectively. Since $F_{\rho, D}-F_{\rho, E}=F_{D}-F_{E}=D_{0}-E_{0}$, we get

$$
\frac{L(s, \rho, D) / L\left(1-s, \rho^{-1}, D\right)}{L(s, \rho, E) / L\left(1-s, \rho^{-1}, E\right)}=\rho(D-E) \cdot|D-E|^{1 / 2-s} .
$$

Now we note that $\rho(D-E)=\rho(x-E)$, solve for $L(s, \rho, D)$, multiply by $\delta_{x}(D)|D|^{-w}$, and sum over $D$. The result follows.

Remark 2.7. In $\S 4$, we shall need another form of the factors in Theorem 2.6. Let us write $E=$ $E_{0}+2 E_{1}$, with $E_{0}$ effective and square-free. Let $F_{0}=F_{\rho, E}-E_{0}$ be the part of $F_{\rho, E}$ supported on $S$, and let $F_{1}=\sum_{v} v$, the sum over $v \in S-S_{\rho, E}$. It follows from Lemma 2.4 that

$$
\begin{aligned}
& \frac{L(s, \rho, E)}{L\left(1-s, \rho^{-1}, E\right)} \cdot \rho(x-E)|E|^{s-1 / 2} \\
& \quad=\epsilon\left(\rho \chi_{E}\right) \rho\left(x-E_{0}\right) q^{g-1}\left|F_{0}\right|^{1 / 2}\left|F_{1}\right|\left(q^{-s}\right)^{2 g-2+\operatorname{deg}\left(F_{0}+F_{1}\right)} \prod_{v \in S-S_{\rho, E}} \frac{1-\rho \chi_{E}(v)|v|^{-s}}{|v|^{1-s}-\rho^{-1} \chi_{E}(v)} .
\end{aligned}
$$

Note that $0 \leqslant F_{0}+F_{1} \leqslant F$, and that the product has finite, non-zero limits as $\operatorname{Re}(s) \rightarrow \pm \infty$.

\section{The second functional equation}

The second functional equation, Theorem 3.3, describes the action of $V(F, n)$ under the involution $(s, w) \rightarrow(w, s)$. The first step, Lemma 3.1, describes the double Dirichlet series in terms of a double sum that is symmetric except for the factor $\chi_{D_{2}}\left(D_{1}\right)$. We then analyze the relation between $\chi_{D_{1}}\left(D_{2}\right)$ and $\chi_{D_{2}}\left(D_{1}\right)$ for two effective divisors $D_{1}, D_{2} \in \operatorname{Div}(C-S)$. This relation, given in Lemma 3.2, follows from quadratic reciprocity and the definitions in $\S 1$. Once we have these two lemmas, the proof of Theorem 3.3 is easily obtained.

LEMma 3.1. If $\rho_{1}$ and $\rho_{2}$ are characters of $X(F, n)$ and $\operatorname{Re}(s), \operatorname{Re}(w)>1$, then

$$
Z\left(s, w ; \rho_{1}, \rho_{2}\right)=L\left(2 s+2 w-1, \rho_{1}{ }^{2} \rho_{2}^{2} ; C-S\right) \sum_{D_{1}, D_{2}} \frac{\rho_{1}\left(D_{1}\right) \rho_{2}\left(D_{2}\right)}{\left|D_{1}\right|^{s}\left|D_{2}\right|^{w}} \chi_{D_{2}}\left(D_{1}\right),
$$

where the sum is over disjoint, effective divisors in $\operatorname{Div}(C-S)$.

Proof. All divisors below are effective and in $\operatorname{Div}(C-S)$, with additional conditions as noted. In the region we are considering, all sums under consideration converge absolutely, so we can rearrange at will. Write $Z\left(s, w ; \rho_{1}, \rho_{2}\right)$ as a sum over $D_{2}$, and use (1.3) and (1.2) to get

$$
\begin{aligned}
Z\left(s, w ; \rho_{1}, \rho_{2}\right) & =\sum_{D_{2}} \rho_{2}\left(D_{2}\right)\left|D_{2}\right|^{-w} L\left(s, \rho_{1} \chi_{D_{2}} ; C-S-S_{D_{2}}\right) a\left(s, \rho_{1}, D_{2}\right) \\
& =\sum_{D_{2}} \frac{\rho_{2}\left(D_{2}\right)}{\left|D_{2}\right|^{w}} \sum_{D_{1}} \frac{\rho_{1} \chi_{D_{2}}\left(D_{1}\right)}{\left|D_{1}\right|^{s}} \sum_{2 d_{1}+2 d_{2} \leqslant D_{2}} \frac{\mu\left(d_{1}\right) \chi_{D_{2}}\left(d_{1}\right) \rho_{1}\left(d_{1}+2 d_{2}\right)}{\left|d_{1}\right|^{s}\left|d_{2}\right|^{2 s-1}},
\end{aligned}
$$

where $D_{1}$ and $d_{1}$ are disjoint from the conductor of $\chi_{D_{2}}$. Let us write $D_{2}=D_{2}^{\prime}+2 D_{2}^{\prime \prime}$, with $D_{2}^{\prime}$ square-free. Since all these divisors are supported away from $S$, the divisors $D_{1}$ and $d_{1}$ are disjoint from $D_{2}^{\prime}$. The condition $2 d_{1}+2 d_{2} \leqslant D_{2}$ becomes $d_{1}+d_{2} \leqslant D_{2}^{\prime \prime}$.

Next, let us replace $D_{1}$ with a new variable, $D_{1}^{\prime}=D_{1}+d_{1}$. The sum over $D_{1}$ and $d_{1}$ becomes $\sum_{D_{1}^{\prime}, d_{1}} \rho_{1} \chi_{D_{2}}\left(D_{1}^{\prime}\right)\left|D_{1}^{\prime}\right|^{-s} \mu\left(d_{1}\right)$, with $D_{1}^{\prime}$ disjoint from $D_{2}^{\prime}$ and $d_{1} \leqslant D_{1}^{\prime}, d_{1} \leqslant D_{2}^{\prime \prime}-d_{2}$. The sum of $\mu\left(d_{1}\right)$ therefore vanishes unless $D_{1}^{\prime}$ and $D_{2}^{\prime \prime}-d_{2}$ are disjoint, in which case it gives 1 . We now have

$$
Z\left(s, w ; \rho_{1}, \rho_{2}\right)=\sum_{D_{2}=D_{2}^{\prime}+2 D_{2}^{\prime \prime}} \frac{\rho_{2}\left(D_{2}\right)}{\left|D_{2}\right|^{w}} \sum_{d_{2} \leqslant D_{2}^{\prime \prime}} \frac{\rho_{1}\left(2 d_{2}\right)}{\left|d_{2}\right|^{2 s-1}} \sum_{D_{1}^{\prime}} \frac{\rho_{1} \chi_{D_{2}}\left(D_{1}^{\prime}\right)}{\left|D_{1}^{\prime}\right|^{s}},
$$

where $D_{1}^{\prime}$ is disjoint from $D_{2}^{\prime}$ and from $D_{2}^{\prime \prime}-d_{2}$. 


\section{B. Fisher AND S. FriedberG}

Now, replace $D_{2}$ with the new variable $D_{2}^{\prime \prime \prime}=D_{2}-2 d_{2}=D_{2}^{\prime}+2\left(D_{2}^{\prime \prime}-d_{2}\right)$, and note that $\chi_{D_{2}}=\chi_{D_{2}^{\prime \prime \prime}}$. This gives a sum over three variables, $D_{2}^{\prime \prime \prime}, D_{1}^{\prime}$, and $d_{2}$, which are independent except that the first two are disjoint. The sum over $d_{2}$ gives $L\left(2 s+2 w-1, \rho_{1}{ }^{2} \rho_{2}{ }^{2} ; C-S\right)$, and the sum over the other two variables gives the double sum in the statement of the lemma (after dropping the primes from $D_{2}^{\prime \prime \prime}$ and $\left.D_{1}^{\prime}\right)$.

Lemma 3.2. Suppose that $D, D^{\prime} \in \operatorname{Div}(C-S)$ have disjoint supports. Let

$$
\alpha\left(D, D^{\prime}\right)=\chi_{D}\left(D^{\prime}\right) / \chi_{D^{\prime}}(D) .
$$

Then $\alpha\left(D, D^{\prime}\right)$ depends only on the images of $D$ and $D^{\prime}$ in $\operatorname{Pic}_{F}(C) \otimes \mathbb{Z} / 2 \mathbb{Z}$.

We use this lemma to define $\alpha\left(D, D^{\prime}\right)$ even when $D$ and $D^{\prime}$ do not have disjoint supports: choose $D_{1}$ disjoint from $D^{\prime}$ and representing the same class in $\operatorname{Pic}_{F}(C) \otimes \mathbb{Z} / 2 \mathbb{Z}$ as $D$, and define $\alpha\left(D, D^{\prime}\right)=\alpha\left(D_{1}, D^{\prime}\right)$.

Proof. Let $D_{1}=D+(m)+2 G$ with $m \equiv 1(\bmod F)$ and $G \in \operatorname{Div}(C-S)$. Assume, for now, that the support of $D_{1}$ is disjoint from that of $D^{\prime}$. If $T$ is a sufficiently large finite set disjoint from the support

of $D^{\prime}$ and from $S$, then $\operatorname{Div}(T)$ maps onto $\operatorname{Pic}_{F}(C)$. If $G=\left(m_{1}\right)+G_{0}$ with $m_{1} \equiv 1 \bmod F$ and $G_{0} \in$ $\operatorname{Div}(T)$ then we also have $D_{1}=D+\left(m m_{1}^{2}\right)+2 G_{0}$. We may thus assume that both $(m)$ and $G$ have supports disjoint from that of $D^{\prime}$. Then $\chi_{D_{1}}\left(D^{\prime}\right)=\chi_{D}\left(D^{\prime}\right) \chi_{m}\left(D^{\prime}\right)$ and $\chi_{D^{\prime}}\left(D_{1}\right)=\chi_{D^{\prime}}(D) \chi_{D^{\prime}}((m))$. If $D^{\prime}=E^{\prime}+\left(m^{\prime}\right)+2 G^{\prime}$ as in Lemma 1.1 then $D^{\prime}-\left(m_{D^{\prime}}\right)=E^{\prime}-\left(m_{E^{\prime}}\right)+2 G^{\prime} \in \operatorname{Div}(S)+2 \operatorname{Div}(C)$. Since $m \equiv 1(\bmod F)$, this implies that $\chi_{m}\left(D^{\prime}\right)=\chi_{m}\left(\left(m_{D^{\prime}}\right)\right)$. Arguing as above, we can choose $m^{\prime}$ and $G^{\prime}$ so that the support of $\left(m_{D^{\prime}}\right)=\left(m^{\prime}\right)+\left(m_{E^{\prime}}\right)$ is disjoint from that of $(m)$. Then quadratic reciprocity implies that $\chi_{m}\left(D^{\prime}\right)=\chi_{m_{D^{\prime}}}((m))=\chi_{D^{\prime}}((m))$, so $\alpha\left(D, D^{\prime}\right)=\alpha\left(D_{1}, D^{\prime}\right)$ in this case.

Either arguing similarly, or using the symmetry $\alpha\left(D, D^{\prime}\right)=\alpha\left(D^{\prime}, D\right)^{-1}$, it follows that $\alpha\left(D, D^{\prime}\right)$ $=\alpha\left(D, D_{1}^{\prime}\right)$ if $D^{\prime}$ and $D_{1}^{\prime}$ represent the same element of $\operatorname{Pic}_{F}(C) \otimes \mathbb{Z} / 2 \mathbb{Z}$ and both have supports disjoint from that of $D$.

For the general case, suppose that $D_{1}-D, D_{1}^{\prime}-D^{\prime} \in \operatorname{Div}_{P, F}(C-S)+2 \operatorname{Div}(C-S)$ and only assume that $D_{1}$ and $D_{1}^{\prime}$ have disjoint supports. Choose an auxiliary divisor $D_{2}$, with support disjoint from all divisors just mentioned, representing the same class as $D$. From the cases we have already considered, $\alpha\left(D, D^{\prime}\right)=\alpha\left(D_{2}, D^{\prime}\right)=\alpha\left(D_{2}, D_{1}^{\prime}\right)=\alpha\left(D_{1}, D_{1}^{\prime}\right)$.

Theorem 3.3. Let $\eta_{1}$ and $\eta_{2}$ be functions on $X(F, n)$. For $E \in \mathcal{E}$, let $\delta_{E}$ denote the characteristic function $\delta_{E}(D)=1$ if $D$ and $E$ represent the same class in $\operatorname{Pic}_{F}(C) \otimes \mathbb{Z} / 2 \mathbb{Z}, \delta_{E}(D)=0$ otherwise. Assume that $n$ is even, so that $\delta_{E}$ can be thought of as a function on $X(F, n)$. Assume that $\operatorname{Re}(s)$, $\operatorname{Re}(w)>1$. Then

$$
Z\left(s, w ; \eta_{1}, \eta_{2}\right)=\sum_{E_{1}, E_{2} \in \mathcal{E}} \alpha\left(E_{2}, E_{1}\right) Z\left(w, s ; \delta_{E_{2}} \eta_{2}, \delta_{E_{1}} \eta_{1}\right)
$$

In particular, for any $x, y \in X(F, n)$,

$$
Z\left(s, w ; \delta_{x}, \delta_{y}\right)=\alpha(x, y) Z\left(w, s ; \delta_{y}, \delta_{x}\right) .
$$

Proof. For the final statement, note that for $x \in X(F, n)$, there is only one value of $E \in \mathcal{E}$ for which $\delta_{E} \delta_{x} \neq 0$. For the general statement, it is enough to consider the case that $\eta_{1}$ and $\eta_{2}$ are characters, by linearity. We will write $\rho_{j}$ instead of $\eta_{j}$ from now on.

We start with the result of Lemma 3.1. Replace $\chi_{D_{2}}\left(D_{1}\right)$ with $\alpha\left(D_{2}, D_{1}\right) \chi_{D_{1}}\left(D_{2}\right)$ and multiply by $1=\sum_{E_{1}, E_{2} \in \mathcal{E}} \delta_{E_{1}}\left(D_{1}\right) \delta_{E_{2}}\left(D_{2}\right)$ :

$$
\begin{aligned}
Z\left(s, w ; \rho_{1}, \rho_{2}\right)= & L\left(2 s+2 w-1, \rho_{1}{ }^{2} \rho_{2}{ }^{2} ; C-S\right) \\
& \times \sum_{E_{1}, E_{2} \in \mathcal{E}} \sum_{D_{1}, D_{2}} \frac{\delta_{E_{1}} \rho_{1}\left(D_{1}\right) \delta_{E_{2}} \rho_{2}\left(D_{2}\right)}{\left|D_{1}\right|^{s}\left|D_{2}\right|^{w}} \alpha\left(D_{2}, D_{1}\right) \chi_{D_{1}}\left(D_{2}\right),
\end{aligned}
$$




\section{Double DiRICHLET SERIES OVER FUNCTION FIELDS}

where the inner sum is over disjoint, effective divisors $D_{1}, D_{2} \in \operatorname{Div}(C-S)$. Because of the factor $\delta_{E_{1}}\left(D_{1}\right) \delta_{E_{2}}\left(D_{2}\right)$, and Lemma 3.2, we can replace $\alpha\left(D_{2}, D_{1}\right)$ with $\alpha\left(E_{2}, E_{1}\right)$ and move it out of the inner sum.

Next, express $\delta_{E_{1}}$ and $\delta_{E_{2}}$ as sums of characters: $\delta_{E_{j}}=(1 / \bar{h}) \sum_{\sigma_{j}} \sigma_{j}\left(E_{j}\right)^{-1} \sigma_{j}$, where $\bar{h}$ denotes the order of $\operatorname{Pic}_{F}(C) \otimes \mathbb{Z} / 2 \mathbb{Z}$ and $\sigma_{j}$ runs over the characters of this group. Since $\left(\sigma_{j} \rho_{j}\right)^{2}=\rho_{j}{ }^{2}$, Lemma 3.1 gives

$$
\begin{aligned}
Z\left(s, w ; \rho_{1}, \rho_{2}\right)= & \sum_{E_{1}, E_{2} \in \mathcal{E}} \alpha\left(E_{2}, E_{1}\right) \cdot(1 / \bar{h})^{2} \sum_{\sigma_{1}, \sigma_{2}} \frac{L\left(2 s+2 w-1,\left(\sigma_{1} \rho_{1}\right)^{2}\left(\sigma_{2} \rho_{2}\right)^{2} ; C-S\right)}{\sigma_{1}\left(E_{1}\right) \sigma_{2}\left(E_{2}\right)} \\
& \times \sum_{D_{1}, D_{2}} \frac{\sigma_{1} \rho_{1}\left(D_{1}\right) \sigma_{2} \rho_{2}\left(D_{2}\right)}{\left|D_{1}\right|^{s}\left|D_{2}\right|^{w}} \chi_{D_{1}}\left(D_{2}\right) \\
= & \sum_{E_{1}, E_{2} \in \mathcal{E}} \alpha\left(E_{2}, E_{1}\right) \cdot(1 / \bar{h})^{2} \sum_{\sigma_{1}, \sigma_{2}} \sigma_{1}\left(E_{1}\right)^{-1} \sigma_{2}\left(E_{2}\right)^{-1} Z\left(w, s ; \sigma_{2} \rho_{2}, \sigma_{1} \rho_{1}\right) .
\end{aligned}
$$

The theorem now follows from the definition (1.5) of $Z\left(w, s ; \delta_{E_{2}} \rho_{2}, \delta_{E_{1}} \rho_{1}\right)$.

\section{Proof of the rationality of the double Dirichlet series}

Let $n$ be even, and let

$$
\phi_{n}(s)=1-q^{n(1-s)}, \quad \Phi_{n}(s, w)=\phi_{n}(s) \phi_{n}(w) \phi_{n}\left(s+w-\frac{1}{2}\right) .
$$

The main theorem of this paper is the following.

Theorem 4.1. Let $Z(s, w) \in V(F, n)$ be a double Dirichlet series. Then $Z(s, w)$ has meromorphic continuation to all of $\mathbb{C}^{2}$ and is a rational function in $q^{-s}$ and $q^{-w}$. In fact, $\Phi_{n}(s, w) Z(s, w)$ is a polynomial of degree at most $2 \operatorname{deg}(F)+2 n+4 g-4$ in each of $q^{-s}$ and $q^{-w}$.

Proof. Let $T_{1} \subseteq \mathbb{C}^{2}$ be the tube domain defined by the condition $\operatorname{Re}(w)>\max \left\{1, \frac{3}{2}-\operatorname{Re}(s), \frac{3}{2}-\right.$ $\left.\frac{1}{2} \operatorname{Re}(s)\right\}$. Let $T_{2}$ be the image of $T_{1}$ under the involution $(s, w) \rightarrow(w, s)$, and let $T_{3}$ be the image of $T_{2}$ under the involution $(s, w) \rightarrow\left(1-s, s+w-\frac{1}{2}\right)$. Explicitly, $T_{2}$ is defined by the condition $\operatorname{Re}(s)>$ $\max \left\{1, \frac{3}{2}-\operatorname{Re}(w), \frac{3}{2}-\frac{1}{2} \operatorname{Re}(w)\right\}$ and $T_{3}$ by the conditions $\operatorname{Re}(s)<0$ and $\operatorname{Re}(w)>\max \left\{1, \frac{3}{2}+\operatorname{Re}(s)\right\}$.

Let $Z(s, w) \in V(F, n)$ be given. By Corollary 2.5, the series for $\phi_{n}(s) Z(s, w)$ converges absolutely and uniformly on compacta in $T_{1}$, so it is an analytic function there. The factor $\phi_{n}(s)=\prod_{\omega^{n}=1}(1-$ $\left.\omega q^{1-s}\right)$ here accounts for every possible $\phi\left(s, \rho_{1}\right)$ in Corollary 2.5. Applying the two involutions, $\phi_{n}(w) Z(w, s)$ represents an analytic function on $T_{2}$ and $\phi_{n}\left(s+w-\frac{1}{2}\right) Z\left(s+w-\frac{1}{2}, 1-s\right)$ represents an analytic function on $T_{3}$.

Choose a basis of $V(F, n)$ and let $\mathbf{Z}(s, w)$ be the row vector consisting of basis elements. Then Theorem 3.3 can be expressed as a matrix equation:

$$
\mathbf{Z}(s, w)=\mathbf{Z}(w, s) B,
$$

where $B$ is a matrix of constants and $(s, w) \in T_{1} \cap T_{2}$, i.e. $\operatorname{Re}(s), \operatorname{Re}(w)>1$. Similarly, Theorem 2.6 can be written $\mathbf{Z}(s, w)=\mathbf{Z}\left(1-s, s+w-\frac{1}{2}\right) A\left(q^{-s}\right)$, for $(s, w) \in T_{1}$. Remark 2.7 shows that the entries in $A(t)$ are rational functions in $t$, whose only poles are at $|t|=0$ or $1 / q$. Combine this with (4.1) to obtain

for $(s, w) \in T_{1} \cap T_{3}$.

$$
\mathbf{Z}(s, w)=\mathbf{Z}\left(s+w-\frac{1}{2}, 1-s\right) B A\left(q^{-s}\right)
$$

We can now extend $\mathbf{Z}(s, w)$ to a vector of meromorphic functions on $T_{1} \cup T_{2} \cup T_{3}$, using $\mathbf{Z}(w, s) B$ on $T_{2}$ and $\mathbf{Z}\left(s+w-\frac{1}{2}, 1-s\right) B A\left(q^{-s}\right)$ on $T_{3}$. Since $A\left(q^{-s}\right)$ has no poles in $T_{3}$, the second paragraph of the proof shows that $\Phi_{n}(s, w) \mathbf{Z}(s, w)$ extends to a vector of analytic functions on the tube domain 


\section{B. Fisher AND S. FriedBerG}

$T_{1} \cup T_{2} \cup T_{3}$. The convexity principle for analytic functions on tube domains [Hor90, Theorem 2.5.10] then gives the analytic continuation of this function to all of $\mathbb{C}^{2}$. In particular, (4.2) holds on $\mathbb{C}^{2}$, by the persistence of analytic relations. Applying this relation three times gives

$$
\mathbf{Z}(s, w)=\mathbf{Z}(1-s, 1-w) B A\left(q^{-w}\right) B A\left(q^{1 / 2-s-w}\right) B A\left(q^{-s}\right) .
$$

Now, express $\Phi_{n}(s, w) \mathbf{Z}(s, w)$ in terms of $t=q^{-s}$ and $u=q^{-w}: \Phi_{n}(s, w) \mathbf{Z}(s, w)=\mathbf{P}(t, u)$. Then $\mathbf{P}(t, u)$ is analytic on $(\mathbb{C}-\{0\})^{2}$. For $s$ in a compact set, it is clear that both $\Phi_{n}(s, w)$ and the series for $\mathbf{Z}(s, w)$ converge, absolutely and uniformly, as $\operatorname{Re}(w) \rightarrow \infty$. Similarly, using $\mathbf{Z}(w, s) B$, we get convergence as $\operatorname{Re}(s) \rightarrow \infty$. In terms of $t$ and $u$, this means that we can extend $\mathbf{P}(t, u)$ to $\mathbb{C}^{2}-\{(0,0)\}$. By Hartogs's Extension Theorem [Hor90, Theorem 2.3.2], $\mathbf{P}(t, u)$ extends to an analytic function on all of $\mathbb{C}^{2}$. To show that $\mathbf{P}(t, u)$ is a polynomial of the stated degree it suffices, by Cauchy's integral formula, to show that each entry is $\mathrm{O}\left(|t u|^{2 n+4 g-4+2 \operatorname{deg}(F)}\right)$.

In order to bound $\mathbf{P}(t, u)$, multiply $(4.3)$ by $\Phi_{n}(s, w) \Phi_{n}(1-s, 1-w)$ and express it in terms of $t$ and $u$ :

$$
\Psi_{n}\left(\frac{1}{q t}, \frac{1}{q u}\right) \cdot \mathbf{P}(t, u)=\Psi_{n}(t, u) \cdot \mathbf{P}\left(\frac{1}{q t}, \frac{1}{q u}\right) B A(u) B A(\sqrt{q} t u) B A(t),
$$

where $\Psi_{n}(t, u)=\left(1-q^{n} t^{n}\right)\left(1-\left(q^{3 / 2} t u\right)^{n}\right)\left(1-q^{n} u^{n}\right)$, so that $\Phi_{n}(s, w)=\Psi_{n}\left(q^{-s}, q^{-w}\right)$. As $|t|$, $|u| \rightarrow \infty, \Psi_{n}(t, u)=\mathrm{O}\left(|t u|^{2 n}\right)$, whereas $\Psi_{n}(1 / q t, 1 / q u)$ and $\mathbf{P}(1 / q t, 1 / q u)$ are bounded. Remark 2.7 shows that $A(t)=\mathrm{O}\left(t^{2 g-2+\operatorname{deg}(F)}\right)$, so the factors $A(t), A(u)$, and $A(\sqrt{q} t u)$ contribute at most $|t u|^{4 g-4+2 \operatorname{deg}(F)}$. It follows that $\mathbf{P}(t, u)=\mathrm{O}\left(|t u|^{2 n+4 g-4+2 \operatorname{deg}(F)}\right)$, as claimed.

\section{The size of sums of twisted $L$-functions}

Fix $F, n$ as in $\S 1$, and let $\rho_{1}, \rho_{2} \in \hat{X}(F, n)$. Since by Theorem 4.1 the double Dirichlet series $Z\left(s, w ; \rho_{1}, \rho_{2}\right)$ is a rational function

$$
Z\left(s, w ; \rho_{1}, \rho_{2}\right)=\frac{\sum_{i, j} c_{i, j} q^{-i s} q^{-j w}}{\Phi_{n}(s, w)},
$$

where the coefficients $c_{i, j}$ depend only on $\rho_{1}$ and $\rho_{2}$, one obtains a formula for the sum

$$
\sum_{\substack{0 \leqslant D \in \operatorname{Div}(C-S) \\ \operatorname{deg}(D)=R}} L\left(s, \rho_{1}, D\right) \rho_{2}(D)
$$

simply by taking the coefficient of $q^{-R w}$ in the right-hand side of (5.1). To state this, let $M$ be the degree in $w$ of the numerator of the right-hand side of (5.1); thus $M \leqslant 2 \operatorname{deg}(F)+2 n+4 g-4$. After some manipulations with geometric series one obtains the following proposition.

Proposition 5.1. Let $\rho_{1}, \rho_{2} \in \hat{X}(F, n)$, and suppose that $Z\left(s, w ; \rho_{1}, \rho_{2}\right)$ is given by (5.1). Let $t=q^{-s}$. Fix a congruence class $j \bmod n$, and let

$$
\begin{aligned}
& C_{j}(s)=\left(1-q^{n} t^{n}\right)^{-1}\left(1-q^{n / 2} t^{n}\right)^{-1} \sum_{\substack{i, k \\
k \equiv j \bmod n}} c_{i, k} t^{i} q^{-k}, \\
& D_{j}(s)=\left(1-q^{n} t^{n}\right)^{-1}\left(1-q^{-n / 2} t^{-n}\right)^{-1} \sum_{\substack{i, k \\
k \equiv j \bmod n}} c_{i, k} t^{i}\left(q^{3 / 2} t\right)^{-k} .
\end{aligned}
$$

Then for $R \geqslant M, R \equiv j \bmod n$, one has

$$
\sum_{\substack{0 \leqslant D \in \operatorname{Div}(C-S) \\ \operatorname{deg}(D)=R}} L\left(s, \rho_{1}, D\right) \rho_{2}(D)=C_{j}(s) q^{R}+D_{j}(s)\left(q^{3 / 2} t\right)^{R} .
$$




\section{Double Dirichlet SERIES OVER FUnCTION FIELDS}

In particular, suppose that $\operatorname{Re}(s)>1 / 2$. Then $\left|q^{3 / 2} t\right|<q$. Thus, provided $C_{j}(s) \neq 0$, the sum of $L$-functions times correction factors grows like $C_{j}(s) q^{R}$ as $R \rightarrow \infty$ in the arithmetic progression $R \equiv j \bmod n$.

The problem of estimating sums of twisted $L$-functions was formulated by Goldfeld and Viola [GV79]. Additional results over $\mathbb{Q}$ are due to Jutila [Jut81], Takhtadzhyan and Vinogradov [TV81], and Goldfeld and Hoffstein [GH85]. For a historical survey see the introduction to [GH85]. In the present situation, one should also be able to separate out the square-free divisors and remove the correction factors by carrying out the sieve used by Goldfeld and Hoffstein [GH85, p. 204] and Hoffstein and Rosen [HR92, p. 142]. (Since we have a rational function and are able to compare coefficients, one should be able to avoid much of the analysis such as estimating the contour integral [HR92, p. 146].) However, we do not do so here, since this would take us far from our main focus.

\section{Some examples}

In this section we consider two curves: $\mathbb{P}^{1}$ over $\mathbb{F}_{q}(q$ odd $)$ and the elliptic curve $y^{2}=x^{3}-x-1$ over $\mathbb{F}_{3}$. For the moment, let us work more generally. Suppose that $C$ is a curve over $\mathbb{F}_{q}, q$ odd, such that $\operatorname{Pic}_{0}(C)$ is trivial, i.e. $C$ has class number one. (It is known [LMQ75] that, besides $\mathbb{P}^{1}$ over any finite field $\mathbb{F}_{q}$, there are finitely many such curves.) Suppose also that $C$ has a rational point; choose one, and denote it $\infty$.

Take $F=\infty$, so that $S=\{\infty\}$, and take $n$ to be even. Choose some $B_{1} \in \operatorname{Div}(C-S)$ of degree 1 , so that $B_{1}=\infty-\left(\pi_{\infty}\right)$, for some uniformizing parameter $\pi_{\infty}$. Write $B_{1}=B_{0}+2 G$, with $B_{0}$ effective and square-free. Since $\operatorname{Div}_{P, F}(C-S)=\operatorname{Div}_{0}(C-S)$, the group $X(F, n)$ is isomorphic to $\mathbb{Z} / n \mathbb{Z}$, generated by the image of $B_{1}$. The characters on $X(F, n)$ are all of the form $\omega^{\text {deg }}$, with $\omega^{n}=1$. Similarly, $\operatorname{Pic}_{F}(C)$ is infinite cyclic, generated by the image of $B_{1}$, and $\operatorname{Pic}_{F}(C) \otimes \mathbb{Z} / 2 \mathbb{Z}$ can be identified with $X(F, 2)$. Let us choose $\mathcal{E}=\left\{0, B_{0}\right\}$ as the coset representatives for $\operatorname{Pic}_{F}(C) \otimes \mathbb{Z} / 2 \mathbb{Z}$. If $E=0$ then choose $\chi_{E}$ to be the trivial character, and if $E=B_{0}$ then choose $\chi_{E}=\chi_{\pi_{\infty}}$. With these choices, if $D$ is any divisor in $\operatorname{Div}(C-S)$, and we write $D=(\operatorname{deg} D) \infty+(m)$ with $m$ monic (with respect to $\pi_{\infty}$ ), then $\chi_{D}=\chi_{m}$.

We now turn to the first functional equation, starting with the epsilon-factor. Since $\rho=\omega^{\operatorname{deg}}$ is unramified, only the numerator of $\epsilon\left(\rho \chi_{E}\right)$ depends on $\rho$ (cf. $\left.(2.2)\right)$, so $\epsilon\left(\rho \chi_{E}\right)=\rho\left((\Omega)+F_{\rho, E}\right) \cdot \epsilon\left(\chi_{E}\right)$. Since $\chi_{E}$ is trivial or quadratic, its epsilon-factor is 1 . The degree of $(\Omega)$ is $2 g-2$, and $F_{\rho, E}=F_{E}=0$ if $E=0, F_{\rho, E}=F_{E}=B_{0}+\infty$ if $E=B_{0}$. Thus $\epsilon\left(\rho \chi_{E}\right)=\omega^{2 g-2}$ if $E=0$, and $\epsilon\left(\rho \chi_{E}\right)=\omega^{2 g-1+\operatorname{deg} B_{0}}$ if $E=B_{0}$. Let $\delta_{j}(D)=1$ if $\operatorname{deg} D \equiv j \bmod 2, \delta_{j}(D)=0$ otherwise. Then Theorem 2.6 and Remark 2.7 now give

$$
\frac{Z\left(s, w ; \omega^{\mathrm{deg}}, \delta_{j}\right)}{Z\left(1-s, s+w-\frac{1}{2} ; \omega^{-\operatorname{deg}}, \delta_{j}\right)}=(-\omega)^{2 g-1+j}\left(q^{-s}\right)^{2 g-1} \begin{cases}q^{g} \frac{1-\omega q^{-s}}{1-\omega q^{1-s}} & \text { if } j \equiv 0(\bmod 2), \\ q^{g-1 / 2} & \text { if } j \equiv 1(\bmod 2) .\end{cases}
$$

Next, consider the second functional equation. We start by calculating $\alpha\left(E_{1}, E_{2}\right)$ for $E_{1}, E_{2} \in \mathcal{E}$, as defined in Lemma 3.2. If $E_{1}=0$ or $E_{2}=0$ then $\alpha\left(E_{1}, E_{2}\right)=1$, so let $E_{1}=E_{2}=B_{0}$. In order to compute $\alpha\left(B_{0}, B_{0}\right)$, choose another divisor of degree 1 , say $D^{\prime}=\infty-\left(\pi^{\prime}\right)$, disjoint from $B_{0}+\infty$. Choose $\pi^{\prime}$ to be monic: that is, $\pi^{\prime} / \pi_{\infty} \equiv 1(\bmod \infty)$. Then quadratic reciprocity gives

$$
\alpha\left(B_{0}, B_{0}\right)=\alpha\left(B_{0}, D^{\prime}\right)=\left(\frac{\pi_{\infty}}{\pi^{\prime}}\right)\left(\frac{\pi^{\prime}}{\pi_{\infty}}\right)^{-1}=\left(\frac{-1}{q}\right)=(-1)^{(q-1) / 2} .
$$

Theorem 3.3 now gives

$$
Z\left(s, w ; \delta_{i}, \delta_{j}\right)=\left(\frac{-1}{q}\right)^{i j} Z\left(w, s ; \delta_{j}, \delta_{i}\right)
$$




\section{B. Fisher AND S. FriedberG}

We may regard $Z\left(s, w ; \rho, \delta_{j}\right)$ as a power series in $t=q^{-s}$ and $u=q^{-w}$. By definition, it only involves powers $u^{b}$ with $b \equiv j(\bmod n)$. Furthermore, if $j$ is odd then the first functional equation shows that the coefficient of $u^{b}$ is a polynomial in $t$ of degree at most $b+2 g-1$, and the coefficient of $t^{a}$ in this polynomial determines that of $t^{b+2 g-1-a}$. The same remarks hold for linear combinations of such series, such as $Z\left(s, w ; \delta_{i}, \delta_{j}\right)$. The second functional equation then shows that this series only involves powers $t^{a}$ with $a \equiv i(\bmod n)$; and if $i$ is odd then there is a non-zero coefficient of $t^{a} u^{b}$ only if $b \leqslant a+2 g-1$. Finally, Theorem 4.1 tells us that $\left(1-q^{n} t^{n}\right)\left(1-q^{n} u^{n}\right)\left(1-q^{3 n / 2} t^{n} u^{n}\right) Z\left(s, w ; \delta_{i}, \delta_{j}\right)$ is a polynomial in $t$ and $u$, of degree at most $2 n+4 g-2$ in each variable.

We turn now to specific examples. First let us take $C=\mathbb{P}^{1}$ and $n=2$, and let $\infty$ denote the usual rational point. The remarks above imply that $Z\left(s, w ; \delta_{1}, \delta_{1}\right)=0$, so $Z\left(s, w ; \delta_{0}, \delta_{1}\right)=Z\left(s, w ; 1, \delta_{1}\right)=$ $Z\left(s, w ;(-1)^{\mathrm{deg}}, \delta_{1}\right)$, and the numerator of this rational function has the form $\left(\alpha+\beta t^{2}\right) u$. It is enough to calculate $\alpha=\sum_{D} L(s, 1, D)$, where $D$ runs over the effective divisors of degree 1 . Any such divisor is square-free, and there are no missing Euler factors, so $L(s, 1, D)=L\left(s, \chi_{D} ; \mathbb{P}^{1}-\{\infty\}\right)$. This $L$-series is a polynomial in $t$ of degree $g=0$, so it is equal to its constant term, 1 . Thus $\alpha=q$, which leads to $Z\left(s, w ; 1, \delta_{1}\right) \equiv q u\left(\bmod u^{3}\right)$ and

$$
Z\left(s, w ; \delta_{0}, \delta_{1}\right)=\frac{q u}{\left(1-q^{2} u^{2}\right)\left(1-q^{3} t^{2} u^{2}\right)}=\frac{q\left(1-q^{2} t^{2}\right) u}{\left(1-q^{2} t^{2}\right)\left(1-q^{2} u^{2}\right)\left(1-q^{3} t^{2} u^{2}\right)} .
$$

Next, the second functional equation gives

$$
Z\left(s, w ; \delta_{1}, \delta_{0}\right)=\frac{q t}{\left(1-q^{2} t^{2}\right)\left(1-q^{3} t^{2} u^{2}\right)}=\frac{q t\left(1-q^{2} u^{2}\right)}{\left(1-q^{2} t^{2}\right)\left(1-q^{2} u^{2}\right)\left(1-q^{3} t^{2} u^{2}\right)} .
$$

The first functional equation for this series gives a linear combination of $Z\left(1-s, s+w-\frac{1}{2} ; \delta_{0}, \delta_{0}\right)$ and $Z\left(1-s, s+w-\frac{1}{2} ; \delta_{1}, \delta_{0}\right)$; solving for the former (a moderate calculation) leads to

$$
Z\left(s, w ; \delta_{0}, \delta_{0}\right)=\frac{1-q^{4} t^{2} u^{2}}{\left(1-q^{2} t^{2}\right)\left(1-q^{2} u^{2}\right)\left(1-q^{3} t^{2} u^{2}\right)} .
$$

Adding these up yields

$$
Z(s, w ; 1,1)=\frac{1+q t+q u-q^{3} t^{2} u-q^{3} t u^{2}-q^{4} t^{2} u^{2}}{\left(1-q^{2} t^{2}\right)\left(1-q^{2} u^{2}\right)\left(1-q^{3} t^{2} u^{2}\right)}=\frac{1-q^{2} t u}{(1-q t)(1-q u)\left(1-q^{3} t^{2} u^{2}\right)} .
$$

By Remark 1.5, this single power series determines $Z\left(s, w ; \delta_{i}, \delta_{j}\right)$ for any even $n$ and all $i$ and $j$.

Applying Proposition 5.1 to $Z(s, w ; 1,1)$ gives, for $R \geqslant 2$,

$$
\sum_{\operatorname{deg}(D)=R} L(s, 1, D)= \begin{cases}\frac{1+2 q t-q^{2} t^{2}}{\left(1-q^{2} t^{2}\right)\left(1-q t^{2}\right)} q^{R}-\frac{q t+\left(q-q^{2}\right) t^{2}+q^{2} t^{3}}{\left(1-q^{2} t^{2}\right)\left(1-q t^{2}\right)}\left(q^{3 / 2} t\right)^{R} & \text { if } R \equiv 0 \bmod 2, \\ \frac{1}{\left(1-q t^{2}\right)} q^{R}-\frac{\sqrt{q} t}{\left(1-q t^{2}\right)}\left(q^{3 / 2} t\right)^{R} & \text { if } R \equiv 1 \bmod 2,\end{cases}
$$

where $t=q^{-s}$, and the sum is over effective divisors supported on $\mathbb{A}^{1}=\mathbb{P}^{1}-\{\infty\}$. If we write $D=(m)+R \infty$ then we can think of this as a sum over monic $m \in \mathbb{F}_{q}[x]$ of degree $R$.

Next we turn to the case that $C$ is an elliptic curve (genus 1 with a rational point). Then $\operatorname{Pic}_{0}(C) \cong C\left(\mathbb{F}_{q}\right)$. Since the number, $N$, of rational points satisfies $|1+q-N| \leqslant 2 \sqrt{q}$, we can have $N=1$ only if $q \leqslant 4$. Since we are avoiding characteristic 2 (for quadratic characters) we take for our second example $n=2$ and $q=3$, and $C$ given by $y^{2}=x^{3}-x-1$. This affine Weierstrass equation has no rational points, so $C\left(\mathbb{F}_{q}\right)=\{\infty\}$. The second functional equation implies that $Z\left(s, w ; \delta_{1}, \delta_{1}\right)=0$ again, so $Z\left(s, w ; \delta_{0}, \delta_{1}\right)=Z\left(s, w ; 1, \delta_{1}\right)=Z\left(s, w ;(-1)^{\mathrm{deg}}, \delta_{1}\right)$ as before. This time, the numerator of this rational function has degree at most 6 in $t$ and $u$.

In order to compute $Z\left(s, w ; 1, \delta_{1}\right)$ it suffices to compute this up to terms in $t$ and $u$ of degree 6 . Since only odd powers of $u$ occur, we only need the coefficients of $u^{1}, u^{3}$, and $u^{5}$. This does not 


\section{Double Dirichlet SERIES OVER FUNCTION FIELDS}

allow room for any square factors (since there are no rational points except $\infty$ ) so the correction terms do not enter into this calculation. The coefficient of $u^{b}$ is a polynomial in $t$ of degree at most $b+1$. Using the two functional equations, it suffices to calculate the coefficients of $t^{0} u^{1}, t^{0} u^{3}, t^{2} u^{3}$, $t^{0} u^{5}$, and $t^{2} u^{5}$.

An effective divisor of degree $d$ on $C-S$ has the form $D=(f)+d \infty$, where $\operatorname{ord}_{\infty}(f)=-d$ and $f$ has no other poles. Since we are interested in the case $d \leqslant 5$, we can take $f=a_{5} x y+a_{4} x^{2}+a_{3} y+$ $a_{2} x+a_{0}$, where $x$ and $y$ are the coordinate functions on the elliptic curve, and the coefficients lie in $\mathbb{F}_{q}$. Then $\chi_{D}\left(D^{\prime}\right)=\left(\frac{f}{D^{\prime}}\right)$, and it is convenient to interpret this as zero if $f \equiv 0\left(\bmod D^{\prime}\right)$. (For the degrees we are considering, this comes up only when $\operatorname{deg} D^{\prime}=2$ and $\operatorname{deg} D=5$.)

It is not hard now to compute $Z\left(s, w ; 1, \delta_{1}\right)$. As before, the two functional equations then give $Z\left(s, w ; \delta_{1}, \delta_{0}\right)$ and $Z\left(s, w ; \delta_{0}, \delta_{0}\right)$. Here are the results:

$$
\begin{aligned}
Z\left(s, w ; \delta_{0}, \delta_{1}\right)= & \frac{\left[q^{2}-q^{2} t^{2}+q^{4} t^{4}\right] u^{3}+\left[\left(q^{4}-q^{5}\right) t^{2}+\left(q^{5}-q^{6}\right) t^{4}\right] u^{5}}{\left(1-q^{2} u^{2}\right)\left(1-q^{3} t^{2} u^{2}\right)}, \\
Z\left(s, w ; \delta_{1}, \delta_{0}\right)= & \frac{q^{2} t^{3}+\left[\left(-q^{2}\right) t^{3}+\left(q^{4}-q^{5}\right) t^{5}\right] u^{2}+\left[q^{4} t^{3}+\left(q^{5}-q^{6}\right) t^{5}\right] u^{4}}{\left(1-q^{2} t^{2}\right)\left(1-q^{3} t^{2} u^{2}\right)}, \\
Z\left(s, w ; \delta_{0}, \delta_{0}\right)= & \Phi_{2}(s, w)^{-1}\left(\left[1+\left(q-q^{2}\right) t^{2}\right]\right. \\
& +\left[\left(q-q^{2}\right)+\left(-q+q^{2}-3 q^{3}+q^{4}\right) t^{2}+\left(q^{3}-2 q^{4}+q^{5}\right) t^{4}\right] u^{2} \\
& \left.+\left[\left(q^{3}-2 q^{4}+q^{5}\right) t^{2}+\left(q^{4}-q^{5}+3 q^{6}-q^{7}\right) t^{4}\right] u^{4}-q^{8} t^{6} u^{6}\right) .
\end{aligned}
$$

\section{ACKNOWLEDGEMENTS}

The authors wish to warmly thank Daniel Bump and Jeffrey Hoffstein for helpful conversations and Mike Rosen and David Hayes for helpful email.

\section{REFERENCES}

BFH90a D. Bump, S. Friedberg and J. Hoffstein, Eisenstein series on the metaplectic group and nonvanishing theorems for automorphic L-functions and their derivatives, Ann. of Math. (2) 131 (1990), 53-127.

BFH90b D. Bump, S. Friedberg and J. Hoffstein, Nonvanishing theorems for L-functions of modular forms and their derivatives, Invent. Math. 102 (1990), 543-618.

BFH96 D. Bump, S. Friedberg and J. Hoffstein, On some applications of automorphic forms to number theory, Bull. Amer. Math. Soc. 33 (1996), 157-175.

BFH04 D. Bump, S. Friedberg and J. Hoffstein, Sums of twisted GL(3) automorphic L-functions, in Contributions to automorphic forms, geometry and arithmetic, eds H. Hida, D. Ramakrishnan and F. Shahidi (Johns Hopkins University Press, Baltimore, 2004), in press.

CF67 J. W. S. Cassels and A. Fröhlich (eds), Algebraic number theory (Academic Press, London, 1967).

FH95 S. Friedberg and J. Hoffstein, Nonvanishing theorems for automorphic L-functions on GL(2), Ann. of Math. (2) 142 (1995), 385-423.

FHL03 S. Friedberg, J. Hoffstein and D. Lieman, Double Dirichlet series and the $n$-th order twists of Hecke L-series, Math. Annal. 327 (2003), 315-338.

GH85 D. Goldfeld and J. Hoffstein, Eisenstein series of 1/2-integral weight and the mean value of real Dirichlet L-series, Invent. Math. 80 (1985), 185-208.

GHP82 D. Goldfeld, J. Hoffstein and S. J. Patterson, On automorphic functions of half-integral weight with applications to elliptic curves, Progress in Mathematics, vol. 26 (Birkhäuser, Boston, 1982), 153-193.

GV79 D. Goldfeld and C. Viola, Mean values of L-functions associated to elliptic, Fermat and other curves at the centre of the critical strip, J. Number Theory 11 (1979), 305-320. 


\section{Double DiRIChlet SERIES OVER FUNCTION FIELDS}

Hof93 J. Hoffstein, Eisenstein series and theta functions on the metaplectic group, in Theta functions from the classical to the modern, ed. M. Ram Murty, CRM Proc. \& Lecture Notes 1 (American Mathematical Society, Providence, RI, 1993), 65-104.

HR92 J. Hoffstein and M. Rosen, Average values of L-series in function fields, J. Reine Angew. Math. 426 (1992), 117-150.

Hor90 L. Hörmander, An introduction to complex analysis in several variables, third edition (NorthHolland, Amsterdam, 1990).

Jut81 M. Jutila, On the mean value of $L\left(\frac{1}{2}, \chi\right)$ for real characters, Analysis 1 (1981), 149-161.

LMQ75 J. R. C. Leitzel, M. L. Madan and C. S. Queen, Algebraic function fields with small class number, J. Number Theory 7 (1975), 11-27.

Lie94 D. Lieman, Nonvanishing of L-series associated to cubic twists of elliptic curves, Ann. of Math. (2) 140 (1994), 81-108.

Mor91 C. Moreno, Algebraic curves over finite fields, Cambridge Tracts in Mathematics, vol. 97 (Cambridge University Press, Cambridge, 1991).

Sie56 ～C. L. Siegel, Die Funktionalgleichungen einiger Dirichletscher Reihen, Math. Z. 63 (1956), 363-373.

TV81 L. Takhtadzhyan and A. Vinogradov, Analogues of the Vinogradov-Gauss formula on the critical line, Zap. Nauchn. Sem. Leningrad. Otdel. Mat. Inst. Steklov. (LOMI) 109 (1981), 41-82, 180-181, 182-183.

Wei74 A. Weil, Basic number theory (Springer, New York, 1974).

Benji Fisher benji@member.ams.org

Mathematics Department, Boston College, Chestnut Hill, MA 02467-3806, USA

Solomon Friedberg friedber@bc.edu

Mathematics Department, Boston College, Chestnut Hill, MA 02467-3806, USA 